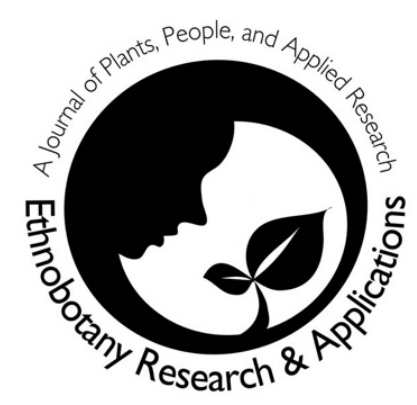

\title{
An ethnopharmacological study of plants used for treatment of diabetes in the Southern and Tribal regions of Khyber Pakhtunkhwa province, Pakistan
}

\author{
Manzoor Ullah, Sultan Mehmood, Maroof Ali, Rainer W. \\ Bussmann, Ali Aldosari, Rehmat Ali Khan, Razi Ullah, \\ Wahid Hussain, Muhammad Abdur Rahman Shah
}

\section{Research}

\begin{abstract}
Background: In the southern and tribal districts of Khyber Pakhtunkhwa reside mainly Pashtun ethnic cultures that preferably use local plants to combat various health issues. We conducted surveys in this terrain to make an inventory of plants used traditionally for the treatment of diabetes.

Methods: A purposive sampling method was applied in the selection of participants, and semi-structured interviews were used for the collection of data. Voucher specimens of each plant species were preserved in the Herbarium Department of Botany University of Science and Technology Bannu, Pakistan.
\end{abstract}

Results: A total of 57 plant species, belonging to 31 plant families, were used to treat diabetes. Among plant parts, leaves were frequently used in the remedies. Similarly, decoction was the most common mode of preparation.

Conclusions: People living in this area commonly use medicinal plants in the traditional medicines to treat diabetes. However, they use such medicines without looking at their potential toxicological effects. Another matter is the immense use of some plants that has created a threat to the loss of their biodiversity in the area, and therefore requires to prioritizes plant resources for conservation and sustainable use.

Key words: Diabetes; ethnobotany; medicinal plants; traditional use; Pakistan.

Correspondence
Manzoor Ullah*
Sultan Mehmood
Maroof Ali
Rainer W. Bussmann
Ali Aldosari
Rehmat Ali Khan
Razi Ullah
Wahid Hussain
Muhammad Abdur Rahman Shah
*Corresponding author:
Department of Botany University of Science \&
Technology Bannu Khyber Pakhtunkhwa, Pakistan
Manzoor Ullah: manzoorkhan536@yahoo.com
Ethnobotany Research \& Applications
18:9 (2019)




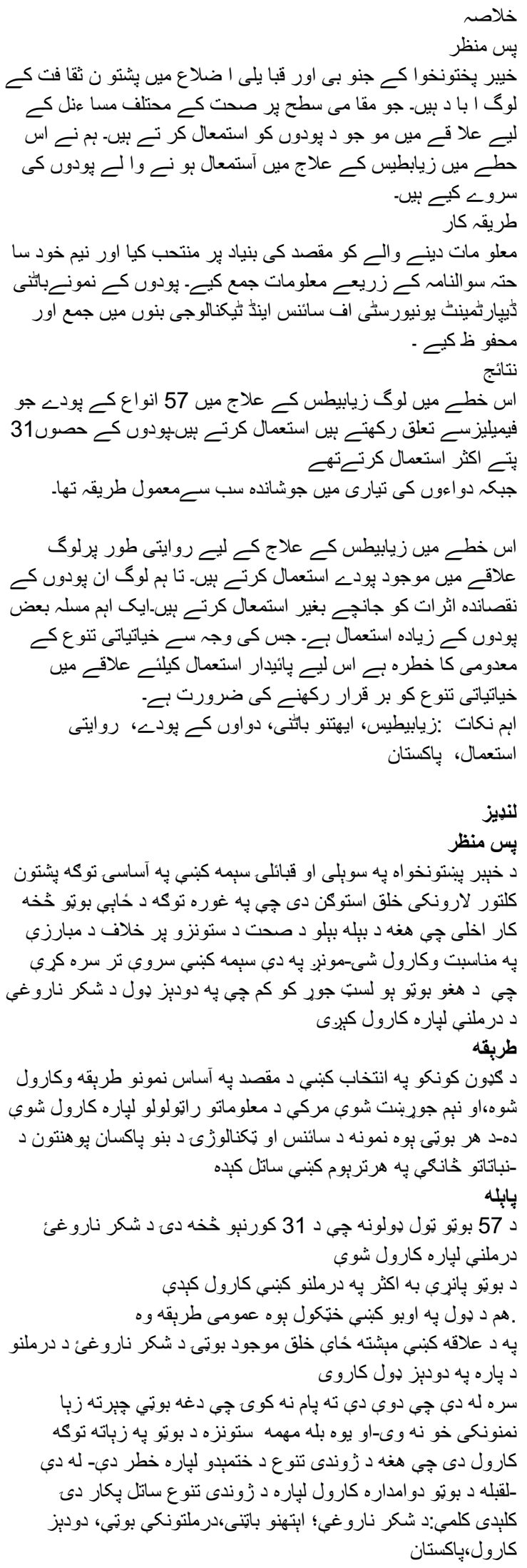

Published: 24 March 2019

\section{Background}

Diabetes is still an incurable health issue that continually increases around the world. A report from the International diabetic federation (IDF) indicates a continuous increase in diabetes prevalence in the last two decades (Ogurtsova et al., 2017). The global diabetes estimate (2010-2030) also points that there will be $69 \%$ increase in number of adults with diabetes in developing countries and $20 \%$ increase in developed countries (Diamond 2011).

People use a variety of antidiabetic medicines that are available in the pharmaceutical market. However, these medicines are expensive, and their side effects are problematic (Adegoke \& Oloyede, 2013; Lo \& Wasser, 2011). A part of modern medicinal research is based on ethnobotanical studies and traditional knowledge and many drugs have been derived from plants. A variety of species are currently undergoing investigation to ascertain their therapeutic efficacy (Torres et al. 2012). Investigations of traditional medicinal plants as alternative therapy are importnat to fight the havoc caused by diabetes. More than 1200 plants are used in the traditional treatment of diabetes (Marles \& Farnsworth 1995; Grover et al. 2002). It is interesting that plant-based medicines for diabetes might still be the most reliable around the world. According to one estimate, $80 \%$ diabetics prefer herbal treatment due to the lesser side effects (Ezuruike \& Prieto 2016). Diabetes management with plant-based medicines is more prevalent in traditional societies of the developing countries, because such medicines are more accessible than the conventional medicines in these societies.

Pakistan is located in the developing world, and diabetes is more prevalent as compared to other Asian countries (Shera et al. 2010). The number of diabetics is estimated to be almost nine million, with almost $11.7 \%$ in the North West Frontier Province (NWFP), now known as Khyber Pakhtunkhwa.

In the southern and tribal districts of Khyber Pakhtunkhwa reside mainly Pashtun ethnic cultures. The terrain is home to the most marginalized, vulnerable, and economically deprived segments of the population. Until recently, access to most of the region was restricted due to conflict. The area has faced harsh economic conditions on account of instability in Afghanistan and operations against terrorists in the area, with huge migration of people. Several ethnobotanical studies from the region show that people utilize local plants in the treatment of diabetes. However, most of these studies (e.g. Gilani et al. 2003; Khan et al. 2011; Khan et al. 2009; Farooq et al. 2012; Qaisar et al. 2013; Hussain et al. 2013; Murad et al. 2013) lack relevant ethnopharmacological information essential for drug discovery purposes. Careful investigation of the traditional medicines is important, as improper remedies, ambiguous products, inappropriate dosage, and side-effects create a potential risk (Robinson \& Zhang 2011). Our study aims to assess informant knowledge about diabetes, enlist and highlight the status of diversity of antidiabetic plants, 
examine the formulation and use of remedies, and assess ethnobotanical and pharmacological literature on the reported antidiabetic plants. We considered it important to carry out research as the indigenous knowledge has come under threat due to migration of the people.

\section{Materials and methods}

\section{Study area}

Khyber Pakhtunkhwa province lies between $34^{\circ} 1^{\prime}$ 33.3012" $\mathrm{N}$ and $71^{\circ} 33^{\prime} 36.4860^{\prime \prime} \mathrm{E}$, with an area of $128961 \mathrm{~km}^{2}$ (Fig. 1). The terrain that extends from Himalaya to Suleiman Mountains shares a $1100 \mathrm{~km}$ border with Afghanistan. It borders Punjab in the east and Baluchistan in the south. Climatic conditions vary in the mountains and plain areas. In the mountain regions, summer remains pleasant while in the plain areas, it is very hot. Freezing temperatures occur in the mountains in winter. The climate in the southern districts is semi-arid with hot summers and mild winters. Autumn and winter are usually dry seasons while summer and spring receive much of the precipitation. The average annual rainfall varies from 600 to $1450 \mathrm{~mm}$ (Wiki, NWFP 2007). The great variability in the regional edaphic conditions, altitude and climatic factors have created a large great range of living places of biodiversity. Hence, biodiversity in this territory reflects a transition zone between Afghan provinces and Punjab and Baluchistan province in Pakistan. This territory is very rich in biodiversity and associated traditional practices.
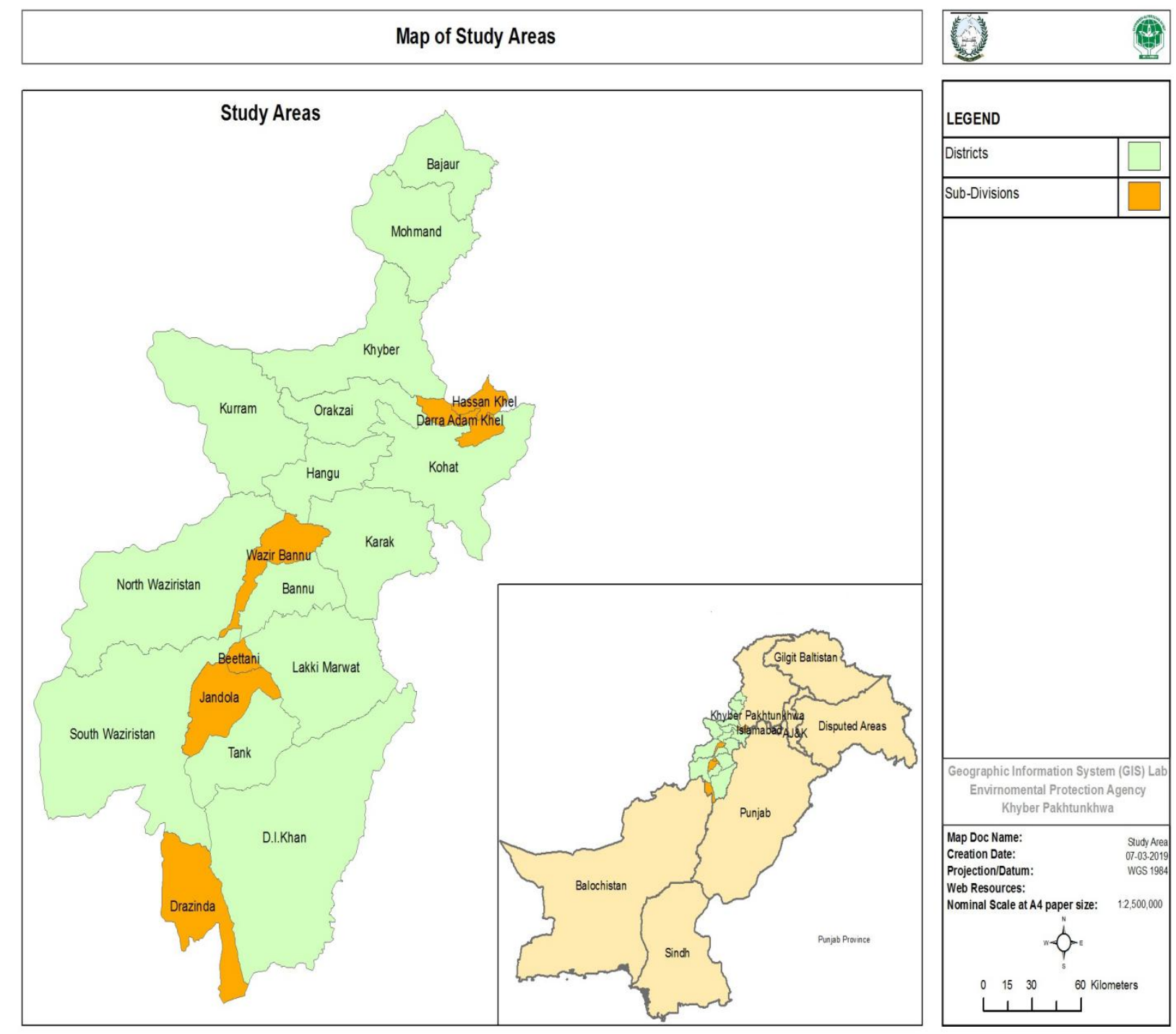

Figure 1. Map of the study area

\section{Socioeconomic background of the study area} The study area comprises fourteen districts and six subdivision of Khyber Pakhtunkhwa province (Fig. $1)$. The main ethnic cultures are Afridi, Bangash, Banochi, Battani, Dawar, Gundapur, Khattak, Kundi,
Mahsud, Marwat, Mohmand, Orakzai, Safi, Seraiki, Sherani, Shinwari, Tarkani, Turi and Wazir. The population are mainly Muslims and some minority religions such as Sikh, Christian, and Hindu. Most people speak the Pashto language while some 
speak other dialects such as Seraiki in Dera Ismail Khan and Tank. The local population in the tribal and frontier regions use Jirga (traditional assemblies) to resolve social problems.

Most of the people in the study area are poor, with very limited facilities. Decades of war and insurgency have wreaked havoc with social structure, economy and infrastructure of the area. Health and education facilities are still limited. Due to limited livelihood opportunities people economically depend on rearing domestic animals, farming, small-scale business, household jobs, recruitment in local security forces, and working on daily wages in the local markets and mining sector. Women along with covering all household duties, take part in farming, collection of fuel wood and carry water.

\section{Data collection and questionnaire}

The present ethnobotanical study was carried out from August 2016 to May 2018, following the Code of Ethics of the International Society of Ethnobiology (ISE, 2006). The first author visited the entire 14 districts and 5 sub-divisions, and 51 communities were selected randomly. A purposive sampling method was employed in selection of traditional healers and elders in which the interviewee assigned the next traditional healers and elders to be included in subsequent interviews. The author received prior informed consent and authorization from local authorities and discussed the research ideas with informants. After obtaining prior informed consent with each participant, antidiabetic plants data were gathered through face-to-face interviews held with participants using semi-structured questionnaires. Interviews were carried out in Pashto language in local dialect. A questionnaire for data collection was designed to address the following information from the informants: knowledge about diabetes, local name of plant, part used and mode of preparation of the herbal medicines. Demographic data of the participants including name, gender, age, age group, location and educational level were also noted (Table1, Annex Questionnaire).

\section{Plant collection and preservation}

The first author collected three fresh samples of each wild plant to create voucher specimens for herbarium deposit. The plant species were collected mostly in flowering stage with assistance of traditional healers and knowledgeable elders. Taxonomists were involved in identification while scientific names and family names of plant species follow (APG IV, 2016). The voucher specimens of the reported plants were prepared, labeled and deposited in the Herbarium of Botany Department University of Science and Technology Bannu.

\section{Data analysis}

Relative frequency of citation (RFC) was calculated as the number of citations (for a given species) divided by the number of all citations for all species (Ocvirk et al. 2013). Plant species that attained higher frequencies were validated by comparing with the available ethnobotanical and pharmacological studies in literature. The literature search was made mainly through Google Scholar, Science direct, PubMed, Scopus and open access journal sources. Table 1. Detail of participants interviewed

\begin{tabular}{|c|c|c|c|c|}
\hline Gender & $\begin{array}{l}\text { Healer } \\
\text { / Elder }\end{array}$ & $\begin{array}{l}\text { Age } \\
\text { group }\end{array}$ & $\begin{array}{l}\text { Education } \\
\text { Level }\end{array}$ & $\begin{array}{l}\text { Education } \\
\text { Level }\end{array}$ \\
\hline \multirow[t]{32}{*}{ Male } & & & & 253 \\
\hline & Elder & & & 168 \\
\hline & & $80-99$ & & 22 \\
\hline & & & Matriculation & 12 \\
\hline & & & Illiterate & 6 \\
\hline & & & Intermediate & 4 \\
\hline & & $60-79$ & & 120 \\
\hline & & & Matriculation & 60 \\
\hline & & & Graduation & 20 \\
\hline & & & Illiterate & 20 \\
\hline & & & Intermediate & 19 \\
\hline & & & Middle & 1 \\
\hline & & $40-59$ & & 26 \\
\hline & & & Illiterate & 11 \\
\hline & & & Matriculation & 8 \\
\hline & & & Intermediate & 5 \\
\hline & & & Graduation & 2 \\
\hline & Healer & & & 85 \\
\hline & & $80-99$ & & 8 \\
\hline & & & Matriculation & 5 \\
\hline & & & Illiterate & 2 \\
\hline & & & Intermediate & 1 \\
\hline & & $60-79$ & & 61 \\
\hline & & & Matriculation & 36 \\
\hline & & & Intermediate & 10 \\
\hline & & & Graduation & 10 \\
\hline & & & Illiterate & 5 \\
\hline & & $40-59$ & & 16 \\
\hline & & & Illiterate & 9 \\
\hline & & & Matriculation & 3 \\
\hline & & & Intermediate & 3 \\
\hline & & & Graduation & 1 \\
\hline \multirow[t]{11}{*}{ Female } & & & & 19 \\
\hline & Elder & & & 19 \\
\hline & & $80-99$ & & 2 \\
\hline & & & Matriculation & 2 \\
\hline & & $60-79$ & & 15 \\
\hline & & & Matriculation & 8 \\
\hline & & & Illiterate & 3 \\
\hline & & & Graduation & 2 \\
\hline & & & Intermediate & 2 \\
\hline & & $40-59$ & & 2 \\
\hline & & & Matriculation & 2 \\
\hline Total & & & & 272 \\
\hline
\end{tabular}




\section{Results}

Informant's assessment on diabetes

Diabetes is commonly known as 'sugar' in all of the regions. The word is proposed because of excessive intake of sugar that leads to the metabolic disorder. The local people showed several views about diabetes. a majority of the healers $(61: 72 \%)$ and elders (112: $60 \%)$ defined that consumption of more sugar as the main cause of diabetes; however, few healers (5: $6 \%)$ and elders (28:15\%) mentioned genetic factors. The remaining healers $19(22 \%)$ and elders $46(25 \%)$ mentioned both genetic factors and consumption more sugar.

The disease was diagnosed through certain symptoms. They diagnosed the diseases e.g. by gathering of ants around urine, slow healing of wounds, high thirst, frequent urination, rough and hard skin, weight loss and weak legs. The inhabitants recommended reducing consumption of sugar as the main way to prevent the disorder. Similarly, some people declared that proper consumption of food items could halt the onset of this disorder. Most of the healers and knowledgeable elders considered exercise as the main way to prevent the severity of diabetes.

\section{Plant species composition}

The participants mentioned 57 plant species of 31 families used for management of diabetes in the study area (Table 2). Among the plant families, the highest number of plant species was reported for Asteraceae (8 species) followed by Lamiaceae (6), Amaryllidaceae (4), and Fabaceae (3). Eight plant families each contributed two species: Apocynaceae, Cucurbitaceae, Euphorbiaceae, Meliaceae, Myrtaceae, Pinaceae, Rhamnaceae and Solanaceae. The remaining 19 families contributed a single species each.
Plant parts used and formulation of remedies

The participants indicated leaves as the most frequently used plant part $(20,27 \%)$ in preparation of remedies followed by fruits $(13,27 \%)$, seeds $(10$, $14 \%)$ and aerial parts (10, 14\%). Stem bark, branches and roots were less frequently used in preparation of recipes (Fig. 2). Decoction with (36) plant species $(40 \%)$ was the common method of remedies preparation. However, seventeen plant parts $(19 \%)$ are used directly mostly fruits. The other forms of formulations are vegetables, powder, juice and infusion (Fig. 3).

\section{Relative frequency citation and use value}

Relative frequency citation was calculated for each plant species (Table 2) and it was found that three species Momordica charantia L. (12.24), Caralluma tuberculata N.E. Br. (10.63) and Citrullus colocynthis (L.) Schrad. (7.84) attained the highest relative frequency of citation. In the other species, a high relative frequency was calculated for Allium cepa $\mathrm{L}$. (4.83), Allium sativum L. (3.76), Withania coagulans (Stocks) Dunal (3.01), Berberis lycium Royle (2.90), Melia azedarach L. (2.79), Trigonella foenumgraecum L. (2.79), Fagonia cretica L. (2.69), Allium ascalonicum L. (2.36), Azadirachta indica A.Juss. (2.36), Syzygium cumini (L.) Skeels (2.36), Ficus benghalensis L. (2.26) and Peganum harmala L. (2.04). The relative importance of plants was evaluated throughthe Use Value index. Momordica charantia L. attained the highest use value (0.419), followed by Caralluma tuberculata N.E. Br. (0.364), Citrullus colocynthis (L.) Schrad. (0.268), Allium cepa L. (0.165), Allium sativum L. (0.129), Withania coagulans (Stocks) Dunal (0.103) etc. (Table 2). The lowest use value was calculated for Euphorbia hirta L. (0.007) and Pinus gerardiana Wall. ex D. Don (0.007).

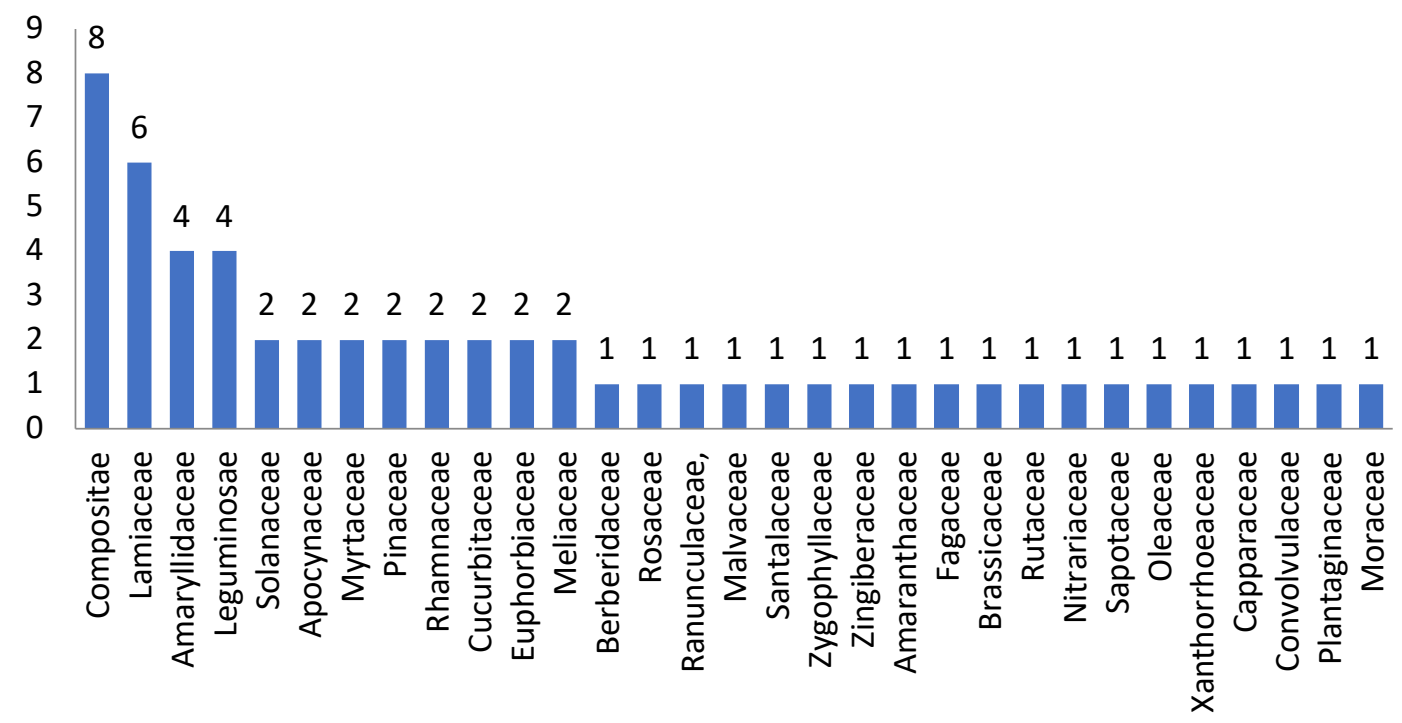

Figure 2. Family distributions of the reported plant species 
Table 2. Antidiabetic plants from Northwest Pakistan

\begin{tabular}{|c|c|c|c|c|c|c|c|}
\hline $\begin{array}{l}\text { Family and } \\
\text { Scientific name }\end{array}$ & $\begin{array}{l}\text { Local } \\
\text { name }\end{array}$ & $\begin{array}{l}\text { Part } \\
\text { used }\end{array}$ & $\begin{array}{l}\text { Life } \\
\text { form }\end{array}$ & $\begin{array}{l}\text { Cultivated } \\
\text { / wild }\end{array}$ & Formulation and use & RFC & UV \\
\hline \multicolumn{8}{|l|}{ AMARANTHACEAE } \\
\hline $\begin{array}{l}\text { Chenopodium murale } \\
\text { L. } \\
\text { (MU-HBD-USTB-19) }\end{array}$ & $\begin{array}{l}\text { Shahkande } \\
\text { r boty/ } \\
\text { Sormy }\end{array}$ & $\begin{array}{l}\text { Whole } \\
\text { plant }\end{array}$ & Herb & Wild & $\begin{array}{l}\text { Cooked leaves or whole } \\
\text { plant decoction is orally } \\
\text { consumed by diabetes. }\end{array}$ & 0.75 & 0.026 \\
\hline \multicolumn{8}{|l|}{ AMARYLLIDACEAE } \\
\hline $\begin{array}{l}\text { Allium ascalonicum L. } \\
\text { (MU-HBD-USTB-06) }\end{array}$ & Sar pyoz & $\begin{array}{l}\text { Bulb, } \\
\text { leaves }\end{array}$ & Herb & Wild & $\begin{array}{l}\text { Cooked bulbs or green } \\
\text { leaves are directly used. }\end{array}$ & 2.36 & 0.081 \\
\hline $\begin{array}{l}\text { Allium carolinianum } \\
\text { DC. } \\
\text { (MU-HBD-USTB-07) }\end{array}$ & $\begin{array}{l}\text { Jangli } \\
\text { wezai }\end{array}$ & Bulb & Herb & Wild & $\begin{array}{l}\text { Cooked bulbs or green } \\
\text { leaves are directly used. }\end{array}$ & 1.93 & 0.066 \\
\hline $\begin{array}{l}\text { Allium cepa L. } \\
\text { (MU-HBD-USTB-08) }\end{array}$ & Pyaz & $\begin{array}{l}\text { Bulb, } \\
\text { leaves }\end{array}$ & Herb & Cultivated & $\begin{array}{l}\text { Leaves are directly used } \\
\text { as raw. Bulb slices are } \\
\text { eaten directly or cooked } \\
\text { as vegetable. }\end{array}$ & 4.83 & 0.165 \\
\hline $\begin{array}{l}\text { Allium sativum L. } \\
\text { (MU-HBD-USTB-09) }\end{array}$ & Woga & Bulbils & Herb & Cultivated & $\begin{array}{l}\text { Leaves are directly used } \\
\text { as raw. Bulb is also eaten } \\
\text { directly or cooked as } \\
\text { vegetable. }\end{array}$ & 3.76 & 0.129 \\
\hline \multicolumn{8}{|l|}{ APOCYNACEAE } \\
\hline $\begin{array}{l}\text { Caralluma tuberculata } \\
\text { N.E.Br. } \\
\text { (MU-HBD-USTB-18) }\end{array}$ & $\begin{array}{l}\text { Pamany or } \\
\text { Pamanky }\end{array}$ & Stem & Herb & Wild & $\begin{array}{l}\text { Stem is cut into pieces } \\
\text { and cooked as vegetable. } \\
\text { Sometimes directly eaten } \\
\text { by diabetes as raw drug. }\end{array}$ & 10.6 & 0.364 \\
\hline $\begin{array}{l}\text { Rhazya stricta Decne } \\
\text { (MU-HBD-USTB-42) }\end{array}$ & Ganderai & Leaves & $\begin{array}{l}\text { Under } \\
\text { shrub }\end{array}$ & Wild & $\begin{array}{l}50 \mathrm{~g} \text { of shade dried leaves } \\
\text { are boiled in } 300 \mathrm{ml} \text { water } \\
\text { to get a decoction. The } \\
\text { decoction is further } \\
\text { diluted to } 500 \mathrm{ml} \text {. }\end{array}$ & 1.61 & 0.055 \\
\hline \multicolumn{8}{|l|}{ ASTERACEAE } \\
\hline $\begin{array}{l}\text { Artemisia absinthium } \\
\text { L. } \\
\text { (MU-HBD-USTB-12) }\end{array}$ & Mastyara & Leaves & Herb & Wild & $\begin{array}{l}\text { Shade dried leaves }(1 \mathrm{~kg}) \\
\text { are boiled in } 5 \mathrm{~L} \text { water to } \\
\text { get a decoction, which is } \\
\text { used in early morning or } \\
\text { before meal one table } \\
\text { spoon per day. }\end{array}$ & 1.40 & 0.048 \\
\hline $\begin{array}{l}\text { Artemisia scoparia } \\
\text { Waldst. \& Kitam. } \\
\text { (MU-HBD-USTB-13) }\end{array}$ & Doorang & $\begin{array}{l}\text { Whole } \\
\text { plant }\end{array}$ & Herb & Wild & $\begin{array}{l}\text { Dried plant material is } \\
\text { boiled in water to get a } \\
\text { decoction and diluted, } \\
\text { which is used before } \\
\text { meal once or two times } \\
\text { per day in a table spoon } \\
\text { amount. }\end{array}$ & 0.64 & 0.022 \\
\hline $\begin{array}{l}\text { Cichorium intybus L. } \\
\text { (MU-HBD-USTB-20) }\end{array}$ & Shin guly & $\begin{array}{l}\text { Leaves, } \\
\text { root } \\
\text { and } \\
\text { stem }\end{array}$ & Herb & Cultivated & $\begin{array}{l}\text { Leaves cooked as } \\
\text { vegetable while decoction } \\
\text { of stem and root }(200 \mathrm{~g}) \\
\text { prepared in } 500 \mathrm{ml} \text { water } \\
\text { is used for diabetes, } \\
\text { hepatitis and malaria. }\end{array}$ & 1.18 & 0.040 \\
\hline $\begin{array}{l}\text { Lactuca sativa L. } \\
\text { (MU-HBD-USTB-30) }\end{array}$ & Saalad & Leaves & Herb & Wild & $\begin{array}{l}\text { Leaves are cooked as } \\
\text { vegetable or eaten raw } \\
\text { with bread. }\end{array}$ & 0.86 & 0.029 \\
\hline $\begin{array}{l}\text { Launaea procumbens } \\
\text { (Roxb.) Ramayya \& } \\
\text { Rajagopal } \\
\text { (MU-HBD-USTB-31) }\end{array}$ & $\begin{array}{l}\text { Shwadi } \\
\text { betai }\end{array}$ & $\begin{array}{l}\text { Aerial } \\
\text { parts }\end{array}$ & Herb & Wild & $\begin{array}{l}\text { Powder of Launaea } \\
\text { procumbens aerial parts } \\
(3 \mathrm{~g}) \text { is put into hot water } \\
\text { glass. The extract is } \\
\text { filtered and a spoon of }\end{array}$ & 0.86 & 0.029 \\
\hline
\end{tabular}




\begin{tabular}{|c|c|c|c|c|c|c|c|}
\hline & & & & & $\begin{array}{l}\text { infusion two times a day } \\
\text { is taken by diabetes. }\end{array}$ & & \\
\hline $\begin{array}{l}\text { Sonchus asper (L.) } \\
\text { Hill } \\
\text { (MU-HBD-USTB-47) }\end{array}$ & Tareza & $\begin{array}{l}\text { Leaves, } \\
\text { Root }\end{array}$ & Herb & Wild & $\begin{array}{l}\text { Infusion of leaves or a } \\
\text { decoction of dried roots is } \\
\text { used. }\end{array}$ & 0.43 & 0.015 \\
\hline $\begin{array}{l}\text { Tanacetum } \\
\text { artemisioides L. } \\
\text { (MU-HBD-USTB-49) }\end{array}$ & Zawil & $\begin{array}{l}\text { Aerial } \\
\text { parts }\end{array}$ & Herb & Wild & $\begin{array}{l}\text { Infusion of } 10 \mathrm{~g} \text { aerial } \\
\text { parts in } 50 \mathrm{ml} \text { is used } \\
\text { prepared and then diluted } \\
\text { to ten part of the original } \\
\text { volume. }\end{array}$ & 0.64 & 0.022 \\
\hline $\begin{array}{l}\text { Taraxacum } \\
\text { campylodes G.E. } \\
\text { Haglund } \\
\text { (MU-HBD-USTB-50) }\end{array}$ & Zer gul & Leaves & Herb & Wild & $\begin{array}{l}\text { Leaves are cooked with } \\
\text { other leafy vegetables. } 1 / 4 \\
\mathrm{~kg} \text { dried aerial parts are } \\
\text { boiled in } 2 \text { liters water } \\
\text { and decoction is filtered } \\
\text { and used for diabetes. } \\
\text { One cup twice a day. }\end{array}$ & 1.07 & 0.037 \\
\hline \multicolumn{8}{|l|}{ CONVOLVULACEAE } \\
\hline $\begin{array}{l}\text { Cuscuta reflexa Roxb } \\
\text { (MU-HBD-USTB-23) }\end{array}$ & $\begin{array}{l}\text { Machi/ } \\
\text { Chambel }\end{array}$ & $\begin{array}{l}\text { Whole } \\
\text { plant }\end{array}$ & Herb & Wild & $\begin{array}{l}\text { Whole plant powder or } \\
\text { decoction is used. }\end{array}$ & 1.50 & 0.051 \\
\hline \multicolumn{8}{|l|}{ CUCURBITACEAE } \\
\hline $\begin{array}{l}\text { Citrullus colocynthis } \\
\text { (L.) Schrad. } \\
\text { (MU-HBD-USTB-21) }\end{array}$ & $\begin{array}{l}\text { Maragonar } \\
\text { ye, } \\
\text { Parpendu, } \\
\text { or tuma }\end{array}$ & Fruit & Herb & Wild & $\begin{array}{l}\text { Fruit powder } 1 \mathrm{~g} \text { per day } \\
\text { or fruit without pericarp is } \\
\text { boiled in water and is } \\
\text { mixed with syrup. Some } \\
\text { peoples mix fruit } \\
\text { decoction with syrup. }\end{array}$ & 7.84 & 0.268 \\
\hline $\begin{array}{l}\text { Momordica charantia } \\
\text { L. } \\
\text { (MU-HBD-USTB-35) }\end{array}$ & Karela & Fruit & Herb & Cultivated & $\begin{array}{l}\text { Fruit slices are first mixed } \\
\text { with salt and then cooked } \\
\text { as vegetable. Some } \\
\text { people prepare juice of } \\
\text { fresh fruit. }\end{array}$ & 12.2 & 0.419 \\
\hline \multicolumn{8}{|l|}{ EUPHORBIACEAE } \\
\hline $\begin{array}{l}\text { Euphorbia hirta L. } \\
\text { (MU-HBD-USTB-26) }\end{array}$ & Chaptary & Leaves & Herb & Wild & $\begin{array}{l}\text { Fresh leaves are crushed } \\
\text { and juice is mixed with } \\
\text { water and is orally taken } \\
\text { for diabetes. }\end{array}$ & 0.21 & 0.007 \\
\hline $\begin{array}{l}\text { Euphorbia prostrata } \\
\text { Aiton } \\
\text { (MU-HBD-USTB-27) }\end{array}$ & $\begin{array}{l}\text { Hara } \\
\text { Chaptary }\end{array}$ & $\begin{array}{l}\text { Aerial } \\
\text { parts }\end{array}$ & Herb & Wild & $\begin{array}{l}\text { A decoction of aerial } \\
\text { parts } 10 \mathrm{~g} \text { prepared in } \\
100 \mathrm{ml} \text { water is used. }\end{array}$ & 0.86 & 0.029 \\
\hline \multicolumn{8}{|l|}{ FABACEAE } \\
\hline $\begin{array}{l}\text { Acacia modesta Wall. } \\
\text { (MU-HBD-USTB-03) }\end{array}$ & Palosa & Leaves & Tree & Wild & $\begin{array}{l}\text { A leaf }(1 / 2 \mathrm{~kg}) \text { is boiled in } \\
\text { water and the decoction } \\
\text { obtained taken two times } \\
\text { a day through table } \\
\text { spoon. Gum is directly } \\
\text { used or treatment of } \\
\text { diabetic. }\end{array}$ & 1.40 & 0.048 \\
\hline $\begin{array}{l}\text { Acacia nilotica (L.) } \\
\text { Delile } \\
\text { (MU-HBD-USTB-04) }\end{array}$ & Kiker & Leaves & Tree & Wild & $\begin{array}{l}\text { Leaves are boiled in } \\
\text { water to get a decoction. }\end{array}$ & 0.86 & 0.029 \\
\hline $\begin{array}{l}\text { Argyrolobium roseum } \\
\text { (Cambess.) Jaub. \& } \\
\text { Spach } \\
\text { (MU-HBD-USTB-11) }\end{array}$ & $\begin{array}{l}\text { Makin } \\
\text { betai }\end{array}$ & $\begin{array}{l}\text { Whole } \\
\text { plant }\end{array}$ & Herb & Wild & $\begin{array}{l}\text { A juice of fresh plant is } \\
\text { used. }\end{array}$ & 0.75 & 0.026 \\
\hline $\begin{array}{l}\text { Trigonella foenum- } \\
\text { graecum L. } \\
\text { (MU-HBD-USTB-52) }\end{array}$ & $\begin{array}{l}\text { Shambreta } \\
\text { / Methi }\end{array}$ & $\begin{array}{l}\text { Aerial } \\
\text { parts } \\
\text { seeds }\end{array}$ & Herb & Wild & $\begin{array}{l}\text { A decoction }(300 \mathrm{~g} / 1 \mathrm{~L} \\
\text { water) of fresh plant is } \\
\text { used two times a day } \\
\text { before meal. The seeds } \\
\text { powder mixed with flour } \\
\text { and sugar is cooked and } \\
\text { used for two months. }\end{array}$ & 2.79 & 0.096 \\
\hline
\end{tabular}




\begin{tabular}{|c|c|c|c|c|c|c|c|}
\hline \multicolumn{8}{|l|}{ FAGACEAE } \\
\hline $\begin{array}{l}\text { Quercus baloot Griff } \\
\text { (MU-HBD-USTB-41) }\end{array}$ & Sayreye & Fruit & Tree & Wild & $\begin{array}{l}\text { A decoction of } 100 \text { dried } \\
\text { fruit in } 500 \text { ml water is } \\
\text { used directly as a raw } \\
\text { drug. }\end{array}$ & 0.64 & 0.022 \\
\hline \multicolumn{8}{|l|}{ LAMIACEAE } \\
\hline $\begin{array}{l}\text { Ajuga integrifolia } \\
\text { Buch. Ham. } \\
\text { (MU-HBD-USTB-05) }\end{array}$ & Soi beetaie & $\begin{array}{l}\text { Whole } \\
\text { plant }\end{array}$ & Herb & Wild & $\begin{array}{l}\text { Crushed plant juice is } \\
\text { taken two times a day } \\
\text { before meal. }\end{array}$ & 0.43 & 0.015 \\
\hline $\begin{array}{l}\text { Ballota } \\
\text { pseudodictamnus (L.) } \\
\text { Benth. } \\
\text { (MU-HBD-USTB-15) }\end{array}$ & Kastoraie & $\begin{array}{l}\text { Aerial } \\
\text { parts }\end{array}$ & Herb & Wild & $\begin{array}{l}\text { A decoction of the aerial } \\
\text { part is used. }\end{array}$ & 0.75 & 0.026 \\
\hline $\begin{array}{l}\text { Marrubium vulgare L. } \\
\text { (MU-HBD-USTB-32) }\end{array}$ & $\begin{array}{l}\text { Dorshol/Bu } \\
\text { taka }\end{array}$ & $\begin{array}{l}\text { Aerial } \\
\text { parts }\end{array}$ & Herb & Wild & $\begin{array}{l}\text { Leaves decoction }(100 \mathrm{~g} / \\
1 \mathrm{~L} \text { water) one table } \\
\text { spoon is taken early in } \\
\text { morning. }\end{array}$ & 0.64 & 0.022 \\
\hline $\begin{array}{l}\text { Mentha longifolia (L.) } \\
\text { L. } \\
\text { (MU-HBD-USTB-34) }\end{array}$ & $\begin{array}{l}\text { Zangli } \\
\text { welany or } \\
\text { podina }\end{array}$ & $\begin{array}{l}\text { Aerial } \\
\text { parts }\end{array}$ & Herb & Wild & $\begin{array}{l}\text { Aerial parts cooked as } \\
\text { vegetable or are boiled in } \\
\text { water to get a decoction. } \\
\text { Some informants } \\
\text { mentioned powder. }\end{array}$ & 0.54 & 0.018 \\
\hline $\begin{array}{l}\text { Salvia reflexa } \\
\text { Hornem. } \\
\text { (MU-HBD-USTB-44) }\end{array}$ & Sugar boti & $\begin{array}{l}\text { Aerial } \\
\text { parts }\end{array}$ & Herb & Wild & $\begin{array}{l}\text { A decoction of shade } \\
\text { dried aerial parts }(10 \mathrm{~g} / \\
250 \mathrm{ml}) \text { is used. }\end{array}$ & 0.75 & 0.026 \\
\hline $\begin{array}{l}\text { Teucrium } \\
\text { stocksianum Boiss. } \\
\text { (MU-HBD-USTB-51) }\end{array}$ & $\begin{array}{l}\text { Harboty/ } \\
\text { Gul bahar }\end{array}$ & $\begin{array}{l}\text { Aerial } \\
\text { parts }\end{array}$ & Herb & Wild & $\begin{array}{l}\text { Fresh or dried aerial parts } \\
\text { are boiled in water to get } \\
\text { a decoction and then } \\
\text { diluted with water. }\end{array}$ & 0.54 & 0.018 \\
\hline \multicolumn{8}{|l|}{ MALVACEAE } \\
\hline $\begin{array}{l}\text { Abelmoschus } \\
\text { moschatus Medik. } \\
\text { (MU-HBD-USTB-01) }\end{array}$ & Bhandi & Fruit & Herb & Cultivated & $\begin{array}{l}\text { Fruit directly eaten as raw } \\
\text { by diabetes or cooked as } \\
\text { vegetable. }\end{array}$ & 0.75 & 0.026 \\
\hline \multicolumn{8}{|l|}{ MELIACEAE } \\
\hline $\begin{array}{l}\text { Azadirachta indica A. } \\
\text { Juss. } \\
\text { (MU-HBD-USTB-14) }\end{array}$ & Neem & $\begin{array}{l}\text { Leaves, } \\
\text { seeds }\end{array}$ & Tree & Wild & $\begin{array}{l}1 \mathrm{Kg} \text { fresh leaves and } \\
\text { seeds in } 5 \mathrm{~L} \text { water is } \\
\text { boiled to get a decoction, } \\
\text { which is taken before } \\
\text { meal two times a day. } \\
\text { Crushed leaves juice is } \\
\text { also used. }\end{array}$ & 2.36 & 0.081 \\
\hline $\begin{array}{l}\text { Melia azedarach L. } \\
\text { (MU-HBD-USTB-33) }\end{array}$ & Bakayan & $\begin{array}{l}\text { Leaves } \\
\text { and } \\
\text { seeds }\end{array}$ & Tree & Wild & $\begin{array}{l}1 \mathrm{Kg} \text { fresh leaves and } \\
\text { seeds in } 5 \mathrm{~L} \text { water is } \\
\text { boiled to get a decoction, } \\
\text { which is taken before } \\
\text { meal two times a day. }\end{array}$ & 2.79 & 0.096 \\
\hline \multicolumn{8}{|l|}{ MORACEAE } \\
\hline $\begin{array}{l}\text { Ficus benghalensis L. } \\
\text { (MU-HBD-USTB-29) }\end{array}$ & Bar & $\begin{array}{l}\text { Bark } \\
\text { and } \\
\text { root }\end{array}$ & Tree & Cultivated & $\begin{array}{l}500 \mathrm{~g} \text { bark or root } \\
\text { decoction prepared in } 1 \mathrm{~L} \\
\text { water is used for } \\
\text { treatment of diabetes. }\end{array}$ & 2.26 & 0.077 \\
\hline \multicolumn{8}{|l|}{ MYRTACEAE } \\
\hline $\begin{array}{l}\text { Eucalyptus globulus } \\
\text { Labill. } \\
\text { (MU-HBD-USTB-25) }\end{array}$ & Safida, L & $\begin{array}{l}\text { Bark } \\
\text { and } \\
\text { leaves }\end{array}$ & Tree & Wild & $\begin{array}{l}\text { Bark and leaves } \\
\text { decoction is considered } \\
\text { useful. }\end{array}$ & 1.40 & 0.048 \\
\hline $\begin{array}{l}\text { Syzygium cumini (L.) } \\
\text { Skeels } \\
\text { (MU-HBD-USTB-48) }\end{array}$ & Jamu & $\begin{array}{l}\text { Fruit, } \\
\text { leaves } \\
\text { and } \\
\text { seeds }\end{array}$ & Tree & Cultivated & $\begin{array}{l}5-10 \text { dry or fresh fruit is } \\
\text { directly used per day and } \\
300 \mathrm{~g} \text { dry or fresh leaves } \\
\text { and seeds decoction is } \\
\text { orally taken to control } \\
\text { diabetic condition. }\end{array}$ & 2.36 & 0.081 \\
\hline
\end{tabular}




\begin{tabular}{|c|c|c|c|c|c|c|c|}
\hline \multicolumn{8}{|l|}{ NITRARIACEAE } \\
\hline $\begin{array}{l}\text { Peganum harmala L. } \\
\text { (MU-HBD-USTB-39) }\end{array}$ & $\begin{array}{l}\text { Spenalai or } \\
\text { sponda }\end{array}$ & Seeds & Herb & Wild & $\begin{array}{l}10 \mathrm{~g} \text { of seeds powder } \\
\text { with a glass of water or } \\
\text { seeds decoction ( } 50 / 400 \\
\mathrm{ml} \text { in water) is taken two } \\
\text { times a day. }\end{array}$ & 2.04 & 0.070 \\
\hline \multicolumn{8}{|l|}{ OLEACEAE } \\
\hline $\begin{array}{l}\text { Olea ferruginea Wall. } \\
\text { ex Aitch. } \\
\text { (MU-HBD-USTB-38) }\end{array}$ & Shwawan & $\begin{array}{l}\text { Branch } \\
\text { es, } \\
\text { leaves } \\
\text { and } \\
\text { seeds }\end{array}$ & Tree & Wild & $\begin{array}{l}\text { Branches, leaves and } \\
\text { seeds are boiled in water } \\
\text { to get a decoction or hot } \\
\text { water infusion of leaves is } \\
\text { used. Fruit powder is also } \\
\text { used. }\end{array}$ & 1.93 & 0.066 \\
\hline \multicolumn{8}{|l|}{ PINACEAE } \\
\hline $\begin{array}{l}\text { Abies pindrow (Royle } \\
\text { ex D. Don) Royle } \\
\text { (MU-HBD-USTB-02) }\end{array}$ & Bejoor & Seeds & Tree & Wild & $\begin{array}{l}\text { A decoction of seeds }(1 / 2 \\
\mathrm{Kg}) \text { prepared in water is } \\
\text { taken orally two time per } \\
\text { day one spoon before } \\
\text { meal. }\end{array}$ & 0.64 & 0.022 \\
\hline $\begin{array}{l}\text { Pinus gerardiana } \\
\text { Wall. ex D. Don } \\
\text { (MU-HBD-USTB-40) }\end{array}$ & Nakhter & Seeds & Tree & Wild & $\begin{array}{l}\text { Seeds are directly } \\
\text { consumed as raw drug. }\end{array}$ & 0.21 & 0.007 \\
\hline \multicolumn{8}{|l|}{ PLANTAGINACEAE } \\
\hline $\begin{array}{l}\text { Nanorrhinum } \\
\text { ramosissimum (Wall.) } \\
\text { Betsche } \\
\text { (MU-HBD-USTB-36) }\end{array}$ & Sanoba & $\begin{array}{l}\text { Whole } \\
\text { plant }\end{array}$ & Herb & Wild & $\begin{array}{l}\text { Dried plant in decoction } \\
\text { or powder is considered } \\
\text { useful in diabetes. }\end{array}$ & 0.64 & 0.022 \\
\hline \multicolumn{8}{|l|}{ RANUNCULACEAE } \\
\hline $\begin{array}{l}\text { Nigella sativa L. } \\
\text { (MU-HBD-USTB-37) }\end{array}$ & Kalwangi & Seeds & Herb & Wild & $\begin{array}{l}200 \mathrm{mg} \text { seeds are used } \\
\text { two times directly or } \\
\text { grinded into powder. }\end{array}$ & 1.83 & 0.063 \\
\hline \multicolumn{8}{|l|}{ RHAMNACEAE } \\
\hline $\begin{array}{l}\text { Ziziphus jajuba Mill. } \\
\text { (MU-HBD-USTB-56) }\end{array}$ & Beer & Fruit & Tree & Wild & $\begin{array}{l}\text { Fruit is directly consumed } \\
\text { as drug. }\end{array}$ & 0.86 & 0.029 \\
\hline $\begin{array}{l}\text { Ziziphus nummularia } \\
\text { (Burm.f.) Wight \& Arn. } \\
\text { (MU-HBD-USTB-57) }\end{array}$ & $\begin{array}{l}\text { Elani/ } \\
\text { karkanra }\end{array}$ & $\begin{array}{l}\text { Whole } \\
\text { plant }\end{array}$ & Shrub & Wild & $\begin{array}{l}\text { A decoction of } 50 \mathrm{~g} \\
\text { leaves in } 300 \mathrm{ml} \text { water is } \\
\text { used. Fresh or dried fruits } \\
\text { are directly used. }\end{array}$ & 0.43 & 0.015 \\
\hline \multicolumn{8}{|l|}{ ROSACEAE } \\
\hline $\begin{array}{l}\text { Rubus vestitus Weihe } \\
\text { (MU-HBD-USTB-43) }\end{array}$ & Kauarch & Fruit & Shrub & Wild & $\begin{array}{l}100 \mathrm{~g} \text { fresh or } 30 \mathrm{~g} \text { dry fruit } \\
\text { per day is directly used. }\end{array}$ & 0.64 & 0.022 \\
\hline \multicolumn{8}{|l|}{ RUTACEAE } \\
\hline $\begin{array}{l}\text { Citrus sinensis (L.) } \\
\text { Osbeck } \\
\text { (MU-HBD-USTB-22) }\end{array}$ & Malta & Fruit & Shrub & Cultivated & $\begin{array}{l}\text { Dried pericarp slices are } \\
\text { cooked in rice for } \\
\text { treatment of diabetes. }\end{array}$ & 0.86 & 0.029 \\
\hline \multicolumn{8}{|l|}{ SANTALACEAE } \\
\hline $\begin{array}{l}\text { Viscum album L. } \\
\text { Wight \& Arn } \\
\text { (MU-HBD-USTB-53) }\end{array}$ & Verai & $\begin{array}{l}\text { Whole } \\
\text { plant }\end{array}$ & Shrub & Wild & $\begin{array}{l}250 \mathrm{~g} \text { of shade dried } \\
\text { whole plant is boiled in } 1 \mathrm{~L} \\
\text { water and the decoction } \\
\text { is taken orally two feeding } \\
\text { spoons a day. }\end{array}$ & 0.75 & 0.026 \\
\hline \multicolumn{8}{|l|}{ SAPOTACEAE } \\
\hline $\begin{array}{l}\text { Sideroxylon } \\
\text { mascatense (A. DC.) } \\
\text { T.D. Penn. } \\
\text { (MU-HBD-USTB-45) }\end{array}$ & Gurgura & $\begin{array}{l}\text { Seeds } \\
\text { and } \\
\text { fruit }\end{array}$ & Shrub & Wild & $\begin{array}{l}2 \mathrm{~g} \text { seeds powder or } 20 \\
\text { dried fruit at night is } \\
\text { considered useful. }\end{array}$ & 0.43 & 0.015 \\
\hline \multicolumn{8}{|l|}{ SOLANACEAE } \\
\hline $\begin{array}{l}\text { Solanum surattense } \\
\text { Burm. f. } \\
\text { (MU-HBD-USTB-46) }\end{array}$ & Marghony & Fruit & Herb & Wild & $\begin{array}{l}2 \mathrm{~g} \text { fruit powder with } \\
\text { sufficient water is taken } \\
\text { before meal two times a } \\
\text { day. }\end{array}$ & 1.18 & 0.040 \\
\hline
\end{tabular}




\begin{tabular}{|c|c|c|c|c|c|c|c|}
\hline $\begin{array}{l}\text { Withania coagulans } \\
\text { (Stocks) Dunal } \\
\text { (MU-HBD-USTB-54) }\end{array}$ & $\begin{array}{l}\text { Hamazeer } \\
\text { a, } \\
\text { shapyanga } \\
\text { or } \\
\text { hafyanga }\end{array}$ & $\begin{array}{l}\text { Fruit } \\
\text { and } \\
\text { leaves }\end{array}$ & $\begin{array}{l}\text { Under } \\
\text { shrub }\end{array}$ & Wild & $\begin{array}{l}\text { Dried fruit powder } 1-3 \mathrm{~g} \text { is } \\
\text { taken before meal. } \\
\text { Overnight infusion of } 10 \mathrm{~g} \\
\text { fruit in } 50 \mathrm{ml} \text { water is } \\
\text { used early in the morning. } \\
\text { A diluted decoction of } \\
\text { shade dried leaves is } \\
\text { taken half of spoon two } \\
\text { times a day. }\end{array}$ & 3.01 & 0.103 \\
\hline \multicolumn{8}{|c|}{ XANTHORROEACEAE } \\
\hline $\begin{array}{l}\text { Aloe vera (L.) Burm. f. } \\
\text { (MU-HBD-USTB-10) }\end{array}$ & $\begin{array}{l}\text { Gurgunyal, } \\
\text { Zargoya }\end{array}$ & Leaves & $\begin{array}{l}\text { Under } \\
\text { shrub }\end{array}$ & Wild & $\begin{array}{l}\text { Leaves are pressed to } \\
\text { get juice or dried leaves } \\
\text { are boiled in water to get } \\
\text { decoction. The juice } \\
\text { mixed with water and } \\
\text { decoction is taken thrice } \\
\text { a day. }\end{array}$ & 1.50 & 0.051 \\
\hline \multicolumn{8}{|l|}{ ZINGIBERACEAE } \\
\hline $\begin{array}{l}\text { Zingiber officinale } \\
\text { Roscoe } \\
\text { (MU-HBD-USTB-55) }\end{array}$ & Adrek & $\begin{array}{l}\text { Rhizom } \\
\text { e }\end{array}$ & Herb & Cultivated & $\begin{array}{l}\text { Powder eaten or put in } \\
\text { water and drink. }\end{array}$ & 0.64 & 0.022 \\
\hline \multicolumn{8}{|l|}{ ZYGOPHYLLACEAE } \\
\hline $\begin{array}{l}\text { Fagonia cretica L. } \\
\text { (MU-HBD-USTB-28) }\end{array}$ & $\begin{array}{l}\text { Spelaghzia } \\
\text {, Azaghai }\end{array}$ & $\begin{array}{l}\text { Aerial } \\
\text { parts }\end{array}$ & Herb & Wild & $\begin{array}{l}\text { Aerial parts are crushed, } \\
\text { or cooked as decoction of } \\
\text { the aerial part is used for } \\
\text { the treatment of diabetes. }\end{array}$ & 2.69 & 0.092 \\
\hline
\end{tabular}

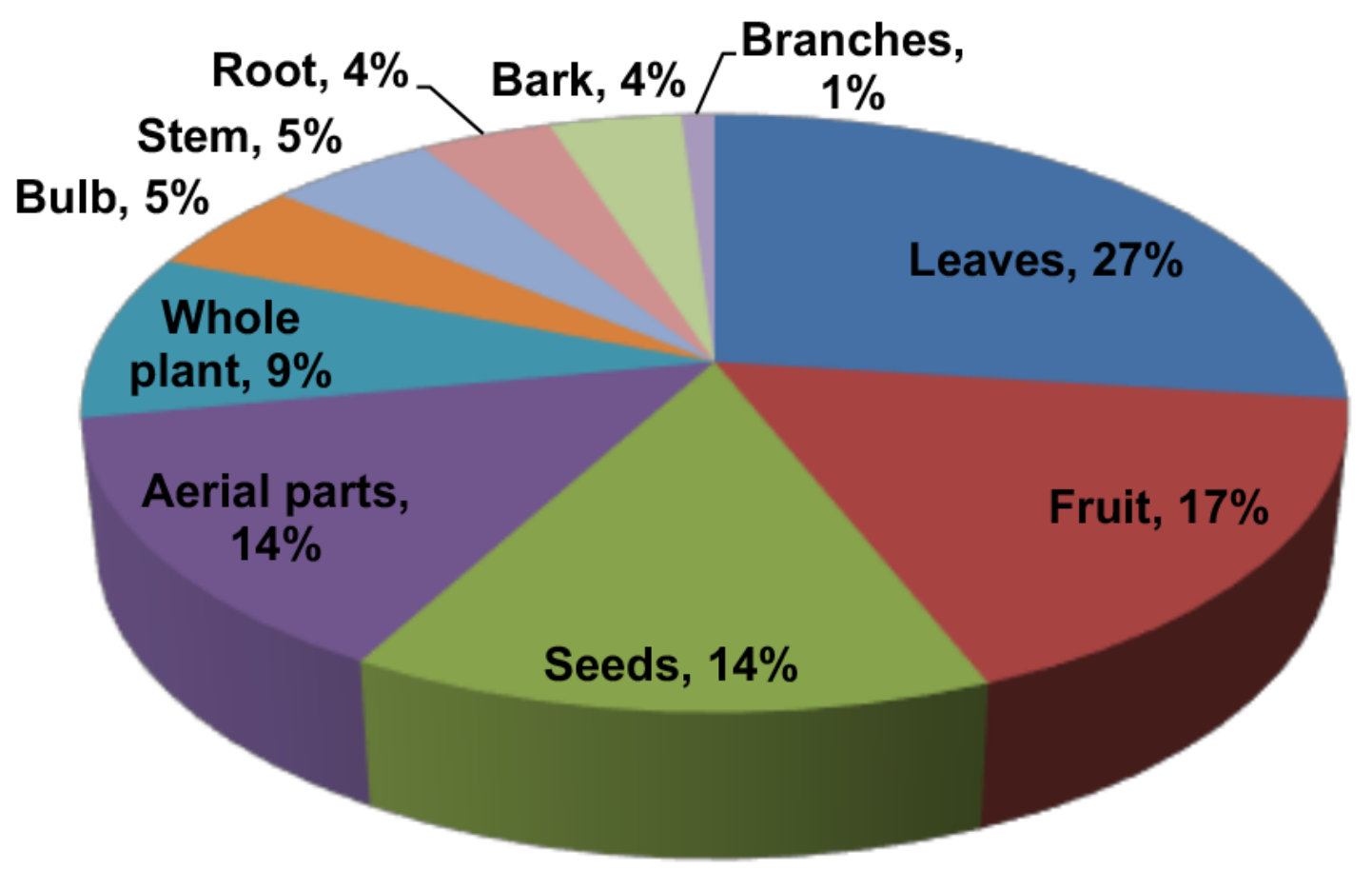

Figure 3. Plant parts used in management of diabetes 


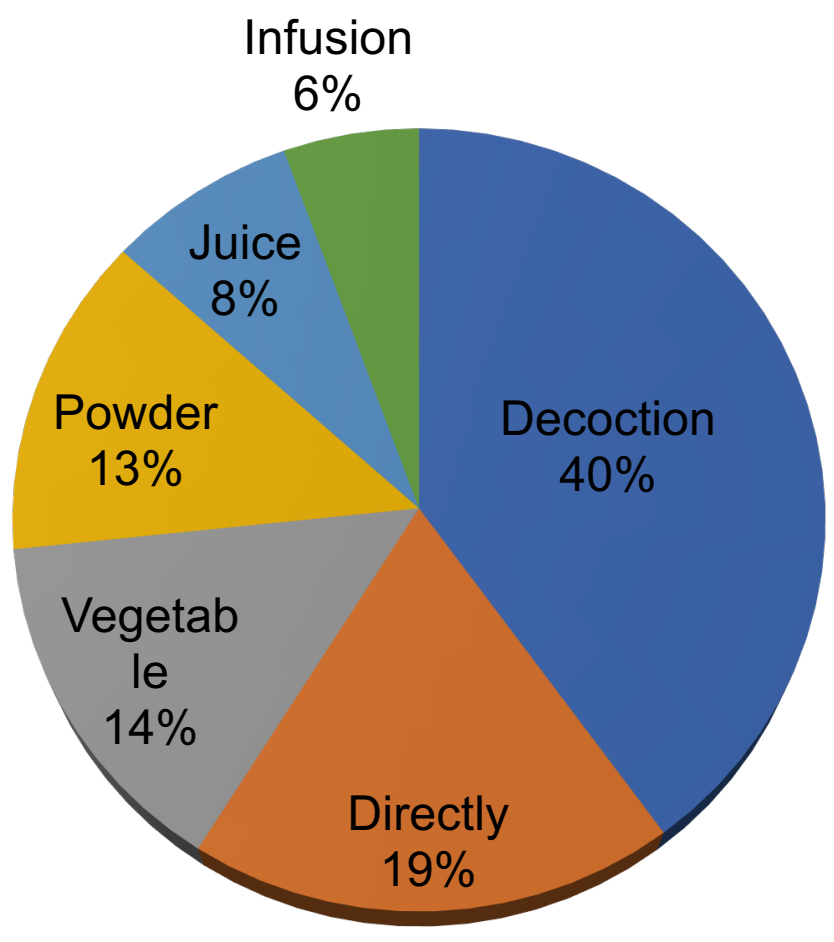

Figure 4. Methods of preparation of remedies

\section{Discussion}

The traditional healers (Hakeems) are the locals that mostly deal in treatment through medicinal plants. While shopkeepers selling herbals products or herbs are called 'Pansars'. The 'Pansars' are not necessarily involved in curing diseases. Many of the elders in the territory are recognized as the persons having greater knowledge after healers.

The traditional healers, knowledgeable elders and locals lay people commonly used the term 'sugar' for diabetes. A majority of the healers $61(72 \%)$ and elders $112(60 \%)$ linked diabetes to consumption of more sugar. Five traditional healers (6\%) and 28 $(15 \%)$ elders mentioned genetic factors as the cause of diabetes. The remaining healers $19(22 \%)$ and elders $46(25 \%)$ mentioned both genetic factors and consumption more sugar. Most of the healers and elders did not employ modern methods for diagnosis of diseases. They diagnosed diseases by certain symptoms as gathering of ants around urine, slow healing of wound, high thirst, frequent urination, rough and hard skin, weight loss and weak legs. The healers $(84 \%)$ recommended the patients to reduce the consumption of sugar in order to prevent the severity of diabetes. The remaining (16\%) declared that proper consumption of food items can halt the onset of this disorder. Most of the healers and knowledgeable elders considered exercise as the main way to prevent the severity caused by diabetes.
The healers were found involved in diagnosis of diabetes patients; and they usually prescribed the available antidiabetic plants for the patients. The elder's belief in the traditional treatment through available plant resource also fortifies the local dependency on herbal remedies for treatment of diabetes. The communities of remote localities were more reliant and confident on traditional antidiabetic plants as compared to communities living around cities. The easy accessibility to synthetic medicines has now greatly affected the communities living around cities. A majority of the inhabitants in the remote communities are however unable to get access to modern health facilities due to poor economic conditions.

The ethnopharmacological information collected from elders in this study showed that much of the indigenous knowledge remains intact with the aged people. The elders disclosed that in time of need plant parts were mostly collected from the wild. The elders also pointed out that preparation of herbal recipes was a time-consuming process, and that the recipes needed preservation. Many residents purchase antidiabetic plants recipes from local market. The elders also stated that most of the residents did not keep antidiabetic plants on account of easy accessibility to traditional healers and modern synthetic medicines. Along these, two main factors responsible for elimination of traditional knowledge from the area were; lack of interest in the 
young generation to use available medicinal plants and that most of the traditional healers kept their knowledge secret (W. Hussain, Badshah, et al. 2018).

The dominance use of the two families Asteraceae and Lamiaceae is linked to their wide distribution and high diversity in the study area, and their activity has been linked to their compound composition (Güzel, Güzelşemme, and Miski 2015; Fortini et al. 2016). Our results agree with the findings of ethnobotanical studies conducted by (Demie, Negash, and Awas 2018) in South-eastern, Ethiopia, (Faruque et al. 2018) in the Bandarban District of Bangladesh and (Barkaoui et al. 2017) in Morocco. This study also specifies that most of the families contributed a single or two species which reflects the diversity of traditional antidiabetic plants in the area.

Among the plant parts leaves, fruit and seeds were also reported as the frequently used plant parts in the ethnobotanical study from the Algerian steppe (Miara et al. 2018). The frequent use of leaves in formulation of recipes has also been reported in the recent ethnobotanical studies (Demie, Negash, and Awas 2018; Faruque et al. 2018; Miara et al. 2018; Barkaoui et al. 2017; Krupa et al. 2018; Tag et al. 2012). This may linked to a higher amount of bioactive compounds in leaves (M. Ullah et al. 2013; Yemele et al. 2015; Ghorbani 2005). The low use of roots in this study could be attributed to unavailability and more laborious job of harvesting, whilst the lesser use of stem bark and branches may relates to frequent use of herbs for treatment of diabetes (Semenya, Potgieter, and Erasmus 2012). In the perspective of conservation of plant species, the use of leaves in preparations of recipes has a more sustainable affect but collection of whole plant may lead to loss of biodiversity from the area. The overexploitation of fruits and seeds may lead to loss of genetic diversity and distribution of species. All the remedies are administered orally, where water is used as solvent in preparation of remedies. Decoction was also a common method of remedies preparation as reported in the ethnobotanical studies (Adeniyi et al. 2018; W. Hussain, Ullah, et al. 2018; Miara et al. 2018; Barkaoui et al. 2017).

The indigenous use of the reported plants was compared with available literature in BioMed Central, Google Scholar, PubMed, PubMed, SCOPUS, and Web of Science. It was found that most of the current study plants have already been reported in the ethnobotanical studies conducted in other parts of the country and around the globe (Table 3). According to the findings of this study six plant taxa; Allium carolinianum DC., Artemisia scoparia Waldst. \& Kitam., Eruca vesicaria (L.) Cav., Salvia reflexa Hornem. Sideroxylon mascatense (A.DC.) T.D. Penn. and Viscum album L. Wight \& Arn were recorded for the first time as antidiabetic agent in comparison to the ethnobotanical literature. The results of this study indicate that on the basis of a single pathology a higher number of plants with accurate information were collected as compared to general ethnobotanical studies conducted in the area. The higher use of some of the wild plants e.g., Withania coagulans and Caralluma tuberculata in the area has created threats to loss of biodiversity from the area. Hence, conservation strategies are necessary to ensure sustainable use of these plants. Scientific validation of medicinal plants based on their traditional use is a very promising approach. Such approach needs careful observation and relevant data such as method of preparation, amount and toxicology of crude drug used. Many plants reported in this study were found having strong antidiabetic activities and several active constituents have been isolated from these plants.

\section{Some details on antidiabetic plants used in the study area \\ Allium cepa L. (Onion)}

A. cepa is an important antidiabetic plant cultivated throughout Pakistan as a culinary agent. The potential of $A$. cepa $\mathrm{L}$. as antidiabetic has been investigated in both human and animal's model. The bulb aqueous extract $(0.4 \mathrm{~g} / 100 \mathrm{~g}$ body weight) significantly decreased blood glucose level by $(70 \%)$, urea $(16 \%)$, creatinine $(32 \%)$ and bilirubin $(28 \%)$ in alloxan induced rats (El-Demerdash, Yousef, and ElNaga 2005). In another similar test in rabbit the aqueous extract at dose 100 and $300 \mathrm{mg} / \mathrm{kg}$ body weight significantly lowered blood glucose level and established the decreased levels of antioxidant enzymes (Ogunmodede et al. 2012). In a human model, the fresh bulb slices at a dose $(100 \mathrm{~g})$ were given to type 1 and 2 diabetic persons. The dose decreases blood glucose level by (50\%) in type 1 diabetic person at 4 hours after administration in comparison to insulin-treated $(70.8 \%)$ diabetic person. The reduction was $20 \%$ in type 2 diabetic person compared to $37.5 \%$ in insulin-treated person (Eldin, Ahmed, and Abd 2010). A diet supplemented with $3 \%$ freeze-dried onion powder was feed to Streptozotocin (STZ) induced rats for 8 weeks that significantly lowered blood glucose and showed strong anti-oxidant potential (Babu and Srinivasan 1997). The isolated sulphur amino acid S-methyl cysteine sulphoxide at a dose of $(200 \mathrm{mg} / \mathrm{kg}$ body weight) for 45 days showed significant hypoglycaemic activity, controlled lipids in serum and tissues and restored the potential of liver hexokinase, glucose 6-phosphatase and 3-Hydroxy3-Methyl-Glutaryl-CoA (HMG CoA) reductase (Kumari, Mathew, and Augusti 1995).

\section{Allium sativum L. (Garlic)}

A. sativum is cultivated throughout Pakistan as an important culinary agent. Its cloves are eaten as raw by diabetes patients to control blood sugar and cholesterol. Its potential as antidiabetic agent was investigated in animal's model. The aqueous extract of bulb at a dose of $(0.4 \mathrm{~g} / 100 \mathrm{~g})$ body weight significantly decreased blood glucose level by $(68 \%)$, urea $(14 \%)$, creatinine $(26 \%)$ and bilirubin $(25 \%)$ in alloxan induced rats (El-Demerdash, Yousef, and ElNaga 2005). In another experiment administration of aqueous extract for one week in alloxan induced diabetes rats significantly decreased blood glucose level (Eyo, Ozougwu, and Echi 2011). In 2006 (Eidi, Eidi, and Esmaeili 2006) evaluated the ethanolic 
extract in STZ induced rats. The extract at a dose of 250 and $500 \mathrm{mg} / \mathrm{kg}$ body weight administration for 14 days produced a dose-dependent decrease in serum glucose, lipid levels, liver function enzyme levels and increased serum insulin levels. The isolated constituent S-allyl cysteine sulphoxide from $A$. sativum has significantly decreased the concentration of serum lipids, blood glucose and activities of serum enzymes like alkaline phosphatase, acid phosphatase, lactate dehydrogenase and liver glucose-6-phosphatase in alloxan induced diabetes rats (Sheela and Augusti 1992).

\section{Aloe vera (L.) Burm.f. (Indian Aloe)}

A well-known traditional medicinal plant $A$. vera is commonly used as wound healing agent in Pakistan. The aerial parts ethanolic extract (100 and 500 $\mathrm{mg} / \mathrm{kg}$ body weight) was employed in normal and hyperglycaemic rats (Afaf, Osman, and Elmahdi 2008). The extract at dose $100 \mathrm{mg} / \mathrm{kg}$ exhibited similar effect to the standard drug glibenclamide in lowering plasma glucose level while highly significant reduction $(P<0.01)$ in plasma glucose level in the group received $500 \mathrm{mg} / \mathrm{kg}$ of the extract. Similarly, dried exudate of leaves at dose of $150 \mathrm{mg} / \mathrm{kg}$ body weight has reduced fasting blood glucose level and improved the levels of the antioxidant enzymes in STZ induced rats (Nwajo 2006). The gelatinous extract from leaves $(350 \mathrm{mg} / \mathrm{kg}$ body weight) containing aloin $(181.7 \mathrm{mg} / \mathrm{g})$ and aloe-emodin (3.6 $\mathrm{mg} / \mathrm{g}$ ) has been investigated in experimentally insulin resistant mice administered for 4 weeks. The extract improved insulin tolerance and fasting blood glucose level (Pérez et al. 2007). In another long term experiment fresh leaf ethanolic extract $(300,500$ $\mathrm{mg} / \mathrm{kg}$ body weight) in alloxan and STZ induced diabetic rats have significantly reduced fasting blood glucose level at day 42 as (44\%) and (73\%), respectively (Shinde, Borkar, and Badwaik 2014).

\section{Azadirachta indica A. Juss. (Neem)}

The most commonly used plant in Pakistan as refrigerant is $A$. indica. Its antidiabetic effect is evident as the leaves ethanolic extract in normal and STZ $(25 \mathrm{mg} / \mathrm{ml})$ induced diabetes rats enhance release of insulin from the pancreas (Chattopadhyay and Bandyopadhyay 2005) while leaves ethanolic extract $(500 \mathrm{mg} / \mathrm{kg})$ in STZ induced diabetes rats decreased blood glucose level and improved pancreatic lesions (Akinola, Caxton-Martins, and Dini 2010).

\section{Berberis lycium Royle (Indian Barberry)}

An important ethnomedicinal plant $B$. lycium root ethanolic extract $(50 \mathrm{mg} / \mathrm{kg}, 100 \mathrm{mg} / \mathrm{kg})$ in alloxanized rats decreased blood glucose from 512 to 396 and 519 to 351, respectively (Gulfraz et al. 2007). In another test, the crude water extract at dose of $(250,500 \mathrm{mg} / \mathrm{kg}$ body weight) significantly reduced blood glucose level in both normal and diabetic rabbits (M. Ahmed and Alamgeer 2009).

\section{Caralluma tuberculata N.E. Br. (Bitter cress)}

Among the wild vegetables, C. tuberculata was the most preferred plant for diabetes. The antidiabetic potential of its aerial parts methanol extract has been tested in STZ induced rats. The extract at dose of $500 \mathrm{mg} / \mathrm{kg}$ body weight/day decreased fasting blood glucose level in hyperglycaemic condition up to $54 \%$ at 4th week with concomitant increase in plasma insulin by $206.8 \%$. A significant decrease in total cholesterol, triglycerides and low density cholesterol levels by $41.5 \%, 36.7 \%$ and $49.1 \%$ have been justified, respectively. An increase of $147.97 \%$ in the cardio-protective lipid high density cholesterol with extract has been observed in comparison to diabetic rat value (Abdel-Sattar et al. 2011).

\section{Citrullus colocynthis (L.) Schrad. (Desert gourd or bitter apple)}

Another important plant C. colocynthis fruit is considered useful remedy. Its fruit pulp extract in STZ induced rats has been found to decrease blood glucose level after 3 and 8 hours and standard drug after 1 st \& 6 th hours, significantly. Insulin levels have also improved at same time intervals (Vinaykumar, Eswarkumar, \& Roy). The saponin fraction from aqueous extract of rind was investigated in normoglycaemic and alloxan induced rabbit (AbdelHassan, Abdel-Barry, and Mohammeda 2000). The fraction with administration of $50 \mathrm{mg} / \mathrm{kg}$ body weight decreased plasma glucose in both types of rabbit. The administration of aqueous extract of leaves at a dose of 250 and $500 \mathrm{mg} / \mathrm{kg}$ body weight significantly reduced blood glucose level from $381 \pm 34$ to $105 \pm$ 35 in alloxanized rats (Gurudeeban and Ramanathan 2010). (Sebbagh et al. 2009) fed STZ induced diabetes rats with $8 \%$ colocynth oil diet. They found significant decrease in plasma glucose levels and restored pancreatic $\beta$-cell mass to normal. In clinical study on 50 type II diabetic patients' 100 $\mathrm{mg}$ fruit capsules or placebos three times a day significantly decreased glycated haemoglobin $(\mathrm{HbA} 1 \mathrm{c})$ and fasting blood glucose level (Huseini et al. 2009).

\section{Fagonia cretica L. (Virgin's Mantle)}

$F$. cretica is a common blood purifying agent in Pakistan and its aerial parts juice or decoction is used for treatment of diabetes. The water extract at dose of $500 \mathrm{mg} / \mathrm{kg}$ was effective in the management of diabetes, causing a $45 \%$ decrease in the plasma glucose level (Nazir et al. 2017).

\section{Melia azedarach L. (Pride of India or chinaberry tree)}

The extract of $M$. azedarach injected intraperitoneally mice showed dose-dependent antidiabetic effects similar to glibenclamide (Seifu et al. 2017). It ethanolic extract in STZ induced diabetes rat significantly decrease blood glucose level (M. F. Khan et al. 2018).

\section{Momordica charantia L. (Bitter gourd)}

$M$. charantia is a common vegetable and recognize as antidiabetic plant in Pakistan. The fresh fruit juice in oral administration test in type 1 diabetic rats at 10 $\mathrm{ml} / \mathrm{kg}$ body weight lowered blood glucose level 
significantly after 30 and 90 minutes by about (30\%) and (10\%) (Matheka et al. 2012).

\section{Rhazya stricta Decne (Harmel)}

The desert plant $R$. stricta fruit and leaves methanol extracts $(80 \%)$ was evaluated in STZ induced mice. The fruit extract has lowered blood glucose level from $(283.34 \pm 5.68)$ to $(219 \pm 77.62)$ in male and from $(344 \pm 31)$ to $(146.00 \pm 40.36)$ in female while leaves extract from $(289.67 \pm 64.36)$ to $(178.34 \pm 17.03)$. Leaves extract has effectively lowered glycosylated hemoglobin to $(6.3 \pm 0.7 \%)$ in male and also reduced total cholesterol content from $(147.88 \pm 21.83 \mathrm{mg} / \mathrm{dl})$ and $(125.89 \pm 14.03 \mathrm{mg} / \mathrm{dl})$ in male and female mice, respectively (A. Ahmed et al. 2015).

\section{Syzygium cumini (L.) Skeels. (Black plum, Java} plum)

Syzygium cumini is an important antidiabetic plant with fruit, leaves and seeds used for treatment of diabetes. The leaves aqueous extract $(25,50,75$ and $100 \mathrm{mg} / \mathrm{ml}$ ) inhibit a-amylase activity by $(60.52 \%), \quad(66.23 \%), \quad(69.33 \%)$ and $(71.71 \%)$ (Sathiavelu et al. 2013). In another experiment barks, leaves, root and seeds significantly $(P<0.001)$ reversed hyperglycaemic activity (Deb et al. 2013). Four active compounds (Lupeol, 12-oleanen-3-ol$3 ß$-acetate, stigmasterol and $ß$-sitosterol) have been isolated from leaves $\mathrm{n}$-hexane fraction (Alam et al. 2012).

\section{Trigonella foenum-graecum L. (Fenugreek)}

$T$. foenum-graecum seeds ethanolic extract $(2 \mathrm{~g} / \mathrm{kg}$, $1 \mathrm{~g} / \mathrm{kg}, 0.5 \mathrm{~g} / \mathrm{kg}$ and $0.1 \mathrm{~g} / \mathrm{kg}$ ) in alloxan induced rats. The extract in diabetic rats significantly $(p<0.05)$ reduced blood glucose by (33.92\%) comparable to that of standard drug, glimepiride $(4 \mathrm{mg} / \mathrm{kg})(35.26 \%)$ (Mowl et al. 2009).

\section{Withania coagulans Dunal (Vegetable rennet, Indian cheese maker)}

$W$. coagulans fruit aqueous extract in STZ induced diabetic rats at a dose of $1 \mathrm{~g} / \mathrm{kg}$ significantly decrease blood glucose, serum lipid peroxidase, cholesterol, hepatic lipid peroxidase level (Hemalatha et al. 2004). The fruit aqueous extract in another experiment at a dose of $1000 \mathrm{mg} / \mathrm{kg}$ has shown maximum fall of $33.2 \%$ in fasting blood glucose after $4 \mathrm{~h}$ and, by $52.9 \%$ on 30th day comparable to glipizide (49.2\%) (Jaiswal, Rai, and Watal 2009). The fruits aqueous extract $(1 \mathrm{~g} / \mathrm{kg}$ body weight) after 7 days demonstrated significant decrease $(p<0.01)$ in the blood glucose by $(52 \%)$, triglycerides, total cholesterol, low density lipid, very low density lipid and very significant increase ( $p<$ 0.01 ) in high density lipids (Hoda et al. 2010). The aqueous extract of flower and root $(150,200 \mathrm{mg} / \mathrm{kg}$ body weight) administration significantly reversed blood glucose and decreased the glycated haemoglobin level by 26 and 44\%, respectively (Bharti et al. 2012).

\section{Toxicology}

Plant based medicines are often considered to be safe and effective agents (George 2011). However, the idea that traditional medicinal products which come from natural sources are completely safe is dangerously false (Calixto 2000). The documentation of medicinal plants and their toxic effects is very essential in the ethnobotanical studies. The locals in the area recognized this problem and were very cautious in selection of antidiabetic plants. Potentially toxic plants used as anti-diabetics in this study were Artemisia scoparia Waldst. \& Kitam., Citrullus colocynthis (L.) Schrad. Euphorbia hirta L. Marrubium vulgare L. Solanum surattense Burm. f., Tanacetum artemisioides L. Teucrium stocksianum Boiss., and Withania coagulans (Stocks) Dunal. The participants stated that preparations of these plants were diluted before use.

\section{Conclusions}

This is the first ethnobotanical study on antidiabetic plants from communities residing in the southern and tribal districts of Khyber Pakhtunkhwa Pakistan. The results indicate that the communities residing in the area have a rich knowledge of antidiabetic plants. The study also highlights that most the plants are collected from wild. Further investigation on the reported plant based on their traditional use is essential. The higher use of some of the wild plants in the area has created threats to loss of biodiversity from the area. Hence, it also prioritizes plant resource for conservation and sustainable use.

\section{Declarations}

Conflict of interest: The authors declare that they have no conflict of interest.

List of Abbreviations:

HbA1c: Glycated haemoglobin

RFC: Relative Frequency of Citation

STZ: Streptozotocin

UV: Use value.

Ethics approval and consent to participate: All participants provided prior oral consent. The study followed the Code of Ethics of the International Society of Ethnobiology (ISE, 2006)

\section{Consent for publication: Not applicable}

Funding: This research work is a part of doctoral research of principal author and claims no funding sources to avail this project.

Author contribution: MU conducted the collection of field data and wrote the initial draft of the manuscript. SM and RAK supervised the project. MA and $\mathrm{WH}$ assisted in the field survey, sampling, and identification of taxon. RU, RB and MAS helped in the data analysis and revision of the manuscript.

\section{Acknowledgments}

We are most grateful to the local authority and chiefs that allowed us to collect indigenous knowledge of antidiabetic plants from the area. We are also 
thankful to the participants for sharing their valuable information.

\author{
Author details: \\ Manzoor Ullah¹, Sultan Mehmood', Maroof Ali $^{2}$, \\ Rainer W. Bussmann ${ }^{3}$, Ali Aldosari ${ }^{4}$, Rehmat Ali \\ Khan $^{5}$, Razi Ullah ${ }^{6}$, Wahid Hussain ${ }^{7}$, Muhammad \\ Abdur Rahman Shah ${ }^{5}$
}

${ }^{1}$ Department of Botany University of Science \& Technology Bannu Khyber Pakhtunkhwa, Pakistan ${ }^{2}$ College of Life Science, Anhui Normal University, Wuhu China 241000.

3llia State University Institute of Botany and Bakuriani Alpine Botanical Garden Department of Ethnobotany, Tbilisi Georgia

${ }^{4}$ Department of Geography, King Saud University, Saudi Arabia

${ }^{5}$ Department of Biotechnology University of Science \& Technology Bannu Khyber Pakhtunkhwa, Pakistan

${ }^{6}$ Institute of Chemical Sciences Gomal University Dera Ismail Khan Khyber Pakhtunkhwa, Pakistan

${ }^{7}$ Department of Botany Government Post Graduate College Parachinar Kurram District Khyber Pakhtunkhwa, Pakistan.

\section{${ }^{*}$ Corresponding author:}

Manzoor Ullah;manzoorkhan536@yahoo.com

\section{Other author emails:}

Sultan Mehmood; dr.sultan@ustb.edu.pk

Maroof Ali; marufturi059@gmail.com

Rainer W. Bussmann;

rainer.bussmann@iliauni.edu.ge

Ali Aldosari; adosari@ksu.edu.sa

Rehmat Ali Khan; rahmatgenetics@gmail.com

Razi Ullah; razijan25@yahoo.com

Wahid Hussain; wahidhussainwahid@gmail.com

Muhammad Abdur Rahman Shah;

abdurrehmanshah16@yahoo.com

\section{Literature Cited}

Abdel-Hassan IA, Abdel-Barry JA, Mohammeda ST. 2000. The hypoglycaemic and antihyperglycaemic effect of Citrullus colocynthis fruit aqueous extract in normal and alloxan diabetic rabbits. Journal of Ethnopharmacology 71: 325-330.

Abdel-Sattar E, Harraz FM, Ghareib SA, Elberry AA, Gabr S, Suliaman MI. 2011. Antihyperglycaemic and hypolipidaemic effects of the methanolic extract of Caralluma tuberculata in streptozotocin-induced diabetic rats. Natural Products Research 25:1 1711179.

Abo KA, Fred-Jaiyesimi AA, Jaiyesimi AEA. 2008. Ethnobotanical studies of medicinal plants used in the management of diabetes mellitus in South
Western Nigeria. Journal of Ethnopharmacology 115: $67-71$.

ADA. 2014. Diagnosis and classification of diabetes mellitus. Diabetes Care. 37:S81-S90.

Adegoke BM, Oloyede OB. 2013. Antihyperglycaemic and antihyperproteinaemic activity of extracts of Picralima nitida seed and Tapinanthus bangwensis leaf on alloxan-induced diabetic rabbits. International Journal of Innovation and Applied Studies. 3: 1125-1131.

Adeniyi A, Asase A, Ekpe PK, Asitoakor BK, AduGyamfi A, Avekor PY. 2018. Ethnobotanical study of medicinal plants from Ghana; confirmation of ethnobotanical uses, and review of biological and toxicological studies on medicinal plants used in Apra Hills Sacred Grove. Journal of Herbal Medicine 14: 76-87.

Afaf IA, Osman MKM, Elmahdi B. 2008. Effect of Aloe vera (Elsabar) Ethanolic Extract on Blood Glucose Level in Wistar Albino Rats.Journal of Applied Sciences Research 4:1841-1845.

Ahmad M, Qureshi R, Arshad M, Khan MA, Zafar M. 2009. Traditional herbal remedies used for the treatment of diabetes from district Attock (Pakistan). Pakistan Journal of Botany. 41:2777-2782.

Ahmad M, Sultana S, Fazl-i-Hadi S, Ben Hadda T, Rashid S, Zafar M, Khan MA, Khan MPZ, Yaseen G. 2014. An Ethnobotanical study of Medicinal Plants in high mountainous region of Chail valley (District Swat-Pakistan). Journal of Ethnobiology and Ethnomedicine 10 (1):36.

Ahmed A, Asad MJ, Ahmad MS, Qureshi R, Shah SI, Gul H, Gulfraz M. 2015. Antidiabetic and hypolipidemic potential of Rhazya stricta Decne extract and its fractions. International Current Pharmaceutical Journal. 4:353-361.

Ahmed M, Alamgeer ST. 2009. A potential adjunct to insulin. Berberis lyceum. Diabetologia Croatica: 38(1), 13-18.

Akgul A, Akgul A, Senol SG, Yildirim H, Secmen O, Dogan Y. 2018. An ethnobotanical study in Midyat (Turkey), a city on the silk road where cultures meet. Journal of Ethnobiology and Ethnomedicine. 14:12.

Akhtar N, Rashid A, Murad W, Bergmeier E. 2013. Diversity and use of ethno-medicinal plants in the region of Swat, North Pakistan. Journal of Ethnobiology and Ethnomedicine. 9:25.

Akinola OB, Caxton-Martins EA, Dini L. 2010. Chronic Treatment with Ethanolic Extract of the Leaves of Azadirachta indica Ameliorates Lesions of Pancreatic Islets in Streptozotocin Diabetes. International Journal of Morphology. 28 (1).

Alam MR, Rahman A Bin, Moniruzzaman M, Kadir MF, Haque MA, Alvi MR-U-H, Ratan MD. 2012. Evaluation of antidiabetic phytochemicals in Syzygium cumini (L.) Skeels (Family: Myrtaceae).Journal of Applied Pharmaceutical Science. 2:94.

Alamgeer TA, Rashid M, Malik MNH, Mushtaq MN. 2013. Ethnomedicinal Survey of plants of Valley Alladand Dehri, Tehsil Batkhela, District Malakand, 
Pakistan. International journal of basic science in medicine. 3 (1) !-10.

Amjad MS, Arshad M, Qureshi R. 2015. Ethnobotanical inventory and folk uses of indigenous plants from Pir Nasoora National Park, Azad Jammu and Kashmir. Asian Pacfic Journal of Tropical Biomedicine. 5:234-241.

APG (2016). An update of the Angiosperm Phylogeny Group classification for the orders and families of flowering plants: APG IV. Botanical Journal of the Linnean Society 181: 1-20.

Babu PS, Srinivasan K. 1997. Hypolipidemic action of curcumin, the active principle of turmeric (Curcuma longa) in streptozotocin induced diabetic rats. Molecular and Cellular Biochemistry 166:169175.

Barkaoui M, Katiri A, Boubaker H, Msanda F. 2017. Ethnobotanical survey of medicinal plants used in the traditional treatment of diabetes in Chtouka Ait Baha and Tiznit (Western Anti-Atlas), Morocco. Journal of Ethnopharmacology. 198:338-350.

Barkatullah IM, Rauf A, Hadda T Ben, Mubarak MS, Patel S. 2015. Quantitative ethnobotanical survey of medicinal flora thriving in Malkand Pass Hills, Khyber PakhtunKhwa Pakistan. Journal of Ethnopharmacology. 169:335-346.

Bharti SK, Kumar A, Sharma NK, Krishnan S, Gupta AK, Padamdeo SR. 2012. Antidiabetic effect of aqueous extract of Withania coagulans flower in Poloxamer-407 induced type 2 diabetic rats. Journal of Medicinal Plants Research. 6:5706-5713.

Bibi T, Ahmad M, Tareen RB, Tareen NM, Jabeen R, Rehman S-U, Sultana S, Zafar M, Yaseen G. 2014. Ethnobotany of medicinal plants in district Mastung of Balochistan province-Pakistan. Journal of Ethnopharmacology. 157:79-89.

Calixto JB. 2000. Efficacy, safety, quality control, marketing and regulatory guidelines for herbal medicines (phytotherapeutic agents). The Brazilian Journal of Medical and Biological Research. 33:179189.

Chattopadhyay RR, Bandyopadhyay M. 2005. Effect of Azadirachta indica leaf extract on serum lipid profile changes in normal and streptozotocin induced diabetic rats. African Journal of biomedical Research. 8:101-104.

Chhetri DR, Parajuli P, Subba GC. 2005. Antidiabetic plants used by Sikkim and Darjeeling Himalayan tribes, India. Journal of Ethnopharmacology. 99:199202.

Deb L, Bhattacharjee C, Shetty SR, Dutta A. 2013. Syzygium cuminii (linn) skeels by reverse pharmacological approaches. Bulletin of Pharmaceutical Research. 3:135-145.

Demie G, Negash M, Awas T. 2018. Ethnobotanical study of medicinal plants used by indigenous people in and around Dirre Sheikh Hussein heritage site of South-eastern Ethiopia. Journal of Ethnopharmacology. 220:87-93.

Diamond J. 2011. Medicine: diabetes in India. Nature. 469:478.
Eidi A, Eidi M, Esmaeili E. 2006. Antidiabetic effect of garlic (Allium sativum L.) in normal and streptozotocin-induced diabetic rats. Phytomedicine. 13:624-629.

El-Demerdash FM, Yousef MI, El-Naga NIA. 2005. Biochemical study on the hypoglycemic effects of onion and garlic in alloxan-induced diabetic rats. Food and Chemical Toxicology. 43:57-63.

Eldin IMT, Ahmed EM, Abd EHM. 2010. Preliminary study of the clinical hypoglycemic effects of Allium cepa (red onion) in type 1 and type 2 diabetic patients. Environmental Health Insights. 4:EHIS5540.

Eyo JE, Ozougwu JC, Echi PC. 2011. Hypoglycaemic effects of Allium cepa, Allium sativum and Zingiber officinale aqueous extracts on alloxan-induced diabetic Rattus novergicus. Medical Journal of Islamic World Academy of Sciences. 2011; 19(3): 121-126

Ezuruike U, Prieto JM. 2016. Assessment of potential herb-drug interactions among Nigerian adults with type-2 diabetes. Frontiers in Pharmacology. 7:248.

Farooq S, Barki A, Yousaf Khan M, Fazal H. 2012. Ethnobotanical studies of the flora of tehsil Birmal in South Waziristan Agency, Pakistan. Pakistan Journal weed Science Research. 18.

Faruque MO, Uddin SB, Barlow JW, Hu S, Dong S, Cai Q, Li X, Hu X. 2018. Quantitative Ethnobotany of Medicinal Plants Used by Indigenous Communities in the Bandarban District of Bangladesh. Frontiers in Pharmacology. 9:40.

Fortini P, Di Marzio P, Guarrera PM, lorizzi M. 2016. Ethnobotanical study on the medicinal plants in the Mainarde Mountains (central-southern Apennine, Italy). Journal of Ethnopharmacology. 184:208-218.

Gbolade AA. 2009. Inventory of antidiabetic plants in selected districts of Lagos State, Nigeria. Journal of Ethnopharmacology. 121:135-139.

George P. 2011. Concerns regarding the safety and toxicity of medicinal plants-An overview. Journal of Applied Pharmaceutical Science. 1:40-44.

Ghorbani A. 2005. Studies on pharmaceutical ethnobotany in the region of Turkmen Sahra, north of Iran:(Part 1): General results. Journal of Ethnopharmacology. 102:58-68.

Gilani SS, Abbas SQ, Shinwari ZK, Hussain F, Nargis K. 2003. Ethnobotanical studies of Kurram Agency, Pakistan through rural community participation. Pakistan Journal Biological Sciences.

Goyal M. 2015. Traditional plants used for the treatment of diabetes mellitus in Sursagar constituency, Jodhpur, Rajasthan-An ethnomedicinal survey. Journal of Ethnopharmacology. 174:364-368.

Grover JK, Yadav S, Vats V. 2002. Medicinal plants of India with anti-diabetic potential.Journal of Ethnopharmacology. 81:81-100.

Gulfraz M, Qadir G, Nosheen F, Parveen Z. 2007. Antihyperglycemic effects of Berberis lyceum Royle 
in alloxan induced diabetic rats. Diabetologia Croatica. 36:49-54.

Gupta S, Sidhu MC, Ahluwalia AS. 2017. Plantbased remedies for the management of diabetes. Current Botany. 8: 34-40.

Gurudeeban S, Ramanathan T. 2010. Antidiabetic effect of Citrullus colocynthis in alloxon-induced diabetic rats. Inventi Rapid Ethnopharmacology. $1: 112$.

Güzel Y, Güzelşemme M, Miski M. 2015. Ethnobotany of medicinal plants used in Antakya: a multicultural district in Hatay Province of Turkey. Journal of Ethnopharmacology. 174:118-152.

Haider M, Zhong L. 2014. Ethno-medicinal uses of plants from district Bahawalpur, Pakistan. Current Research Journal of Biological Sciences. 6:183-190.

Haq F. 2012. The ethno botanical uses of medicinal plants of Allai Valley, Western Himalaya Pakistan. International Journal of Plant Research. 2:21-34.

Haq F, Ahmad H, Alam M. 2011. Traditional uses of medicinal plants of Nandiar Khuwarr catchment (District Battagram), Pakistan.Journal of Medicinal Plants Research. 5:39-48.

Hayat MQ, Khan MA, Ahmad M, Shaheen N, Yasmin G, Akhter S. 2008. Ethnotaxonomical approach in the identification of useful medicinal flora of tehsil Pindigheb (District Attock) Pakistan. Ethnobotany Research and Applications. 6:35-62.

Hemalatha S, Wahi AK, Singh PN, Chansouria JPN. 2004. Hypoglycemic activity of Withania coagulans Dunal in streptozotocin induced diabetic rats. Journal of Ethnopharmacology. 93:261-264.

Hoda Q, Ahmad S, Akhtar M, Najmi AK, Pillai KK, Ahmad SJ. 2010. Antihyperglycaemic and antihyperlipidaemic effect of poly-constituents, in aqueous and chloroform extracts, of Withania coagulans Dunal in experimental type 2 diabetes mellitus in rats. Human \& Experimental Toxicology. 29:653-658.

Hulley IM, Van Wyk B-E. 2018. Quantitative medicinal ethnobotany of Kannaland (western Little Karoo, South Africa): Non-homogeneity amongst villages. South African Journal of Botany doi: 10.1016/j.sajb.2018.03.014.

Huseini HF, Darvishzadeh F, Heshmat R, Jafariazar Z, Raza M, Larijani B. 2009. The clinical investigation of Citrullus colocynthis (L.) schrad fruit in treatment of Type II diabetic patients: a randomized, double blind, placebo-controlled clinical trial. Phytherapy Research. 23:1186-1189.

Hussain K, Nisar MF, Majeed A, Nawaz K, Bhatti KH. 2010. Ethnomedicinal survey for important plants of Jalalpur Jattan, district Gujrat, Punjab, Pakistan. Ethnobotanical Leaflet. 2010:11.

Hussain W, Badshah L, Ullah M, Ali M, Ali A, Hussain F. 2018. Quantitative study of medicinal plants used by the communities residing in Koh-e-Safaid Range, northern Pakistani-Afghan borders. Journal of Ethnobiology Ethnomedicine. 14:30.

Hussain W, Hussain J, Hussain S, khan Shinwari Z, Ali R, Basir A. 2013. Ethono medicinal study of
Parachinar, Kurram Valley (FATA) KPK, Pakistan. Journal of Applied Pharmceutical Sciences. 3:85.

Hussain W, Ullah M, Dastagir G, Badshah LAL. 2018. Quantitative ethnobotanical appraisal of medicinal plants used by inhabitants of lower Kurram, Kurram agency, Pakistan. Avicenna Journal of Phytomedicine.:1-11.

Ibrar M, Rauf A, Hadda T Ben, Mubarak MS, Patel S. 2015. Quantitative ethnobotanical survey of medicinal flora thriving in Malakand Pass Hills, Khyber Pakhtunkhwa, Pakistan. Journal of Ethnopharmacology. 169:335-346.

ljaz F, Iqbal Z, Alam J, Khan SM, Afzal A, Rahman IU, Afzal M, Islam M. 2015. Ethno medicinal study upon folk recipes against various human diseases in Sarban Hills, Abbottabad, Pakistan. World Journal of Zoology. 10:41-46.

Ilyas M, Qureshi R, Shinwari ZK, Arshad M, Mirza SN. 2013. Some ethnoecological aspects of the plants of qalagai hills, kabal valley, swat, Pakistan. International Journal of Agriculture and Biology. 15: (5). 801-810

Ishtiaq M, Mumtaz AS, Hussain T, Ghani A. 2012. Medicinal plant diversity in the flora of Leepa Valley, Muzaffarabad (AJK), Pakistan. African Journal of Biotechnology. 11:3087-3098.

Jaiswal D, Rai PK, Watal G. 2009. Antidiabetic effect of Withania coagulans in experimental rats. Indian Journal Clinical Biochemistry. 24:88-93.

Keter LK, Mutiso PC. 2012. Ethnobotanical studies of medicinal plants used by Traditional Health Practitioners in the management of diabetes in Lower Eastern Province, Kenya. Journal of Ethnopharmacology. 139:74-80.

Khan AA, Ali F, Ihsan M, Hayat K, Nabi G. 2015. Ethnobotanical study of the medicinal plants of Tehsil Charbagh, district Swat, Khyber Pakhtunkhwa, Pakistan. American Eurasian Journal of Agriculture and Environmental Sciences. 15:14641474.

Khan M, Musharaf S, Shinwari ZK. 2011. Ethnobotanical importance of halophytes of Noshpho salt mine, District Karak, Pakistan. Research in Pharmaceutical Biotechnology. 3:46-52.

Khan MF, Rawat AK, Khatoon S, Hussain MK, Mishra A, Negi DS. 2018. In vitro and in vivo Antidiabetic Effect of Extracts of Melia azedarach, Zanthoxylum alatum, and Tanacetum nubigenum.Integrative Medicine Research 7(2):176-83.

Khan SU, Wazir SM, Subhan M, Zahoor M, Kamal M, Taj S. 2009. Some of the ethnobotanically important plants of FR Bannu, NWFP, Pakistan. Pakistan Journal of Plant Sciences. 15:81-85.

Khan SW, Khatoon S. 2008. Ethnobotanical studies on some useful herbs of Haramosh and Bugrote valleys in Gilgit, northern areas of Pakistan. Pakistan Journal of Botany. 40:43.

Krupa J, Sureshkumar J, Silambarasan R, Priyadarshini K, Ayyanar M. 2018. Integration of traditional herbal medicines among the indigenous 
communities in Thiruvarur District of Tamil Nadu, India. Journal of Ayurveda and Integrative Medicine 1-6.

Kumari K, Mathew BC, Augusti KT. 1995. Antidiabetic and hypolipidemic effects of S-methyl cysteine sulfoxide isolated from Allium cepa Linn.Indian Journal of Biochemistry and Biophysics. 32:49-54.

Lo H-C, Wasser SP. 2011. Medicinal mushrooms for glycemic control in diabetes mellitus: history, current status, future perspectives, and unsolved problems. International Journal of Medicinal Mushrooms. 13.

Mahmood A, Mahmood A. 2012. Indigenous wild medicinal plants used by local people of Dudial area, District Mirpur, Azad Jammu and Kashmir, Pakistan. African Journal of. Microbiology Research. 6:38983903.

Mahmood A, Mahmood A, Malik RN. 2012. Indigenous knowledge of medicinal plants from Leepa valley, Azad Jammu and Kashmir, Pakistan. Journal of Ethnopharmacology. 143:338-346.

Mahmood A, Mahmood A, Shaheen H, Qureshi RA, Sangi Y, Gilani SA. 2011. Ethno medicinal survey of plants from district Bhimber Azad Jammu and Kashmir, Pakistan. Journal of Medicinal Plants Research. 5:2348-2360.

Marles RJ, Farnsworth NR. 1995. Antidiabetic plants and their active constituents. Phytomedicine. 2:137189.

Matheka DM, Alkizim FO, Kiama TN, Bukachi F. 2012. Glucose-lowering effects of Momordica charantia (Karela) extract in diabetic rats. African Journal of Pharmacology and Therapeutics. 1(2): 6266.

Miara MD, Bendif H, Hammou MA, Teixidor-Toneu I. 2018. Ethnobotanical survey of medicinal plants used by nomadic peoples in the Algerian steppe. Journal of Ethnopharmacology. 219:248-256.

Mootoosamy A, Mahomoodally MF. 2014. Ethnomedicinal application of native remedies used against diabetes and related complications in Mauritius. Journal of Ethnopharmacology. 151:413444.

Mowl A, Alauddin M, Rahman M, Ahmed K. 2009. Antihyperglycemic effect of Trigonella foenumgraecum (Fenugreek) seed extract in alloxaninduced diabetic rats and its use in diabetes mellitus: a brief qualitative phytochemical and acute toxicity test on the extract. African Journal of Traditional Complement and Alternative Medicines. 6(3):25561.

Murad W, Azizullah A, Adnan M, Tariq A, Khan KU, Waheed S, Ahmad A. 2013. Ethnobotanical assessment of plant resources of Banda Daud Shah, District Karak, Pakistan. Journal of Ethnobiology Ethnomedicine. 9:77.

Nasir S, Ahmed J, Asrar M. 2014. Medicinal Plants: a Promising Resource for Poverty Alleviation in the Milieu of Swat. FUUAST Journal of Biology. 4:237.

Nazir I, ur Rahman N, Alvi Z, Rahman MH, Sendker J, Zhang T, Frankish N, Sheridan H. 2017.
Antidiabetic Activities of an LC/MS Fingerprinted Aqueous Extract of Fagonia cretica L. in Preclinical Models. Planta Medica. 83:1141-1148.

Nwajo HU. 2006. Antioxidant activity of the exudate from Aloe barbadensis leaves in diabetic rats. Biokemistri. 18.

Ocvirk S, Kistler M, Khan S, Talukder SH, Hauner H. 2013. Traditional medicinal plants used for the treatment of diabetes in rural and urban areas of Dhaka, Bangladesh-an ethnobotanical survey. Journal of Ethnobiology Ethnomedicine. 9:43.

Ogunmodede OS, Saalu LC, Ogunlade B, Akunna GG, Oyewopo AO. 2012. An evaluation of the hypoglycemic, antioxidant and hepatoprotective potentials of onion (Allium cepa L.) on alloxaninduced diabetic rabbits. International Journal of Pharmacology. 8:21-29.

Ogurtsova K, da Rocha Fernandes JD, Huang Y, Linnenkamp U, Guariguata L, Cho NH, Cavan D, Shaw JE, Makaroff LE. 2017. IDF Diabetes Atlas: Global estimates for the prevalence of diabetes for 2015 and 2040. Diabetes Research and Clinical Practices. 128:40-50.

Parvaiz M. 2014. Ethnobotanical studies on plant resources of Mangowal, District Gujrat, Punjab, Pakistan. Avicenna Journal of phytomedicine. 4:364.

Pérez YY, Jiménez-Ferrer E, Zamilpa A, HernándezValencia M, Alarcón-Aguilar FJ, Tortoriello J, Román-Ramos R. 2007. Effect of a polyphenol-rich extract from Aloe vera gel on experimentally induced insulin resistance in mice. American Journa of Chinese Medicines. 35:1037-1046.

Qaisar M, Farooq S, Gilani SN, Wasim MA, Kakar M, Shah SWA, Rauf A. 2013. Ethnobotanical survey of medicinal plants used in Wazir and Daur tribes of North Waziristan, Pakistan. Global Veterinaria. 11:285-292.

Qureshi R, Bhatti GR. 2008. Ethnobotany of plants used by the Thari people of Nara Desert, Pakistan. Fitoterapia. 79:468-473.

Qureshi R, Bhatti GR, Memon RA. 2010. Ethnomedicinal uses of herbs from northern part of Nara desert, Pakistan. Pakistan Journal of Botany. 42:839-851.

Qureshi RA, Ghufran MA. 2007. Indigenous knowledge of selected medicinal wild plants of district Attock, Punjab, Pakistan. Pakistan Journal of Botany. 39:2291-2299.

Qureshi RA, Ghufran MA, Sultana KN, Ashraf M, Khan AG. 2007. Ethnomedicinal studies of medicinal plants of Gilgit District and surrounding areas. Ethnobotany Research and Applications. 5:115-122.

Rachid A, Rabah D, Farid L, Zohra SF, Houcine B, Nacéra B. 2012. Ethnopharmacological survey of medicinal plants used in the traditional treatment of diabetes mellitus in the North Western and South Western Algeria. Journal of Medicinal Plants Research. 6:2041-2050.

Rehman K, Khan MA, Ullah Z, Chaudhary HJ. 2015. An ethno botanical perspective of traditional medicinal plants from the Khattak tribe of Chonthra 
Karak, Pakistan.Journal of Ethnopharmacology. 165:251-259.

Robinson MM, Zhang X. 2011. The world medicines situation 2011, traditional medicines: Global situation, issues and challenges. Geneva World Health Organization. 1-4.

Sabeen M, Ahmad SS. 2009. Exploring the folk medicinal flora of Abbotabad city, Pakistan. Ethnobotanical Leaflet. 2009:1.

Sathiavelu A, Sangeetha S, Archit R, Mythili S. 2013. In vitro anti-diabetic activity of aqueous extract of the medicinal plants Nigella sativa, Eugenia jambolana, Andrographis paniculata and Gymnema sylvestre.International Journal of Drug Development and Research. 5:323-328.

Sebbagh N, Cruciani-Guglielmacci C, Ouali F, Berthault M-F, Rouch C, Sari DC, Magnan C. 2009. Comparative effects of Citrullus colocynthis, sunflower and olive oil-enriched diet in streptozotocin-induced diabetes in rats. Diabetes Metabolism. 35:178-184.

Seifu D, Gustafsson LE, Chawla R, Genet S, Debella A, Holst M, Hellström PM. 2017. Antidiabetic and gastric emptying inhibitory effect of herbal Melia azedarach leaf extract in rodent models of diabetes type 2 mellitus. Journal of Experimental Pharmacology. 9:23.

Semenya S, Potgieter M, Erasmus L. 2012. Ethnobotanical survey of medicinal plants used by Bapedi healers to treat diabetes mellitus in the Limpopo Province, South Africa. Journal of Ethnopharmacology. 141:440-445.

Shah A, Marwat SK, Gohar F, Khan A, Bhatti KH, Amin M, Din NU, Ahmad M, Zafar M. 2013. Ethnobotanical study of medicinal plants of semitribal area of Makerwal \& Gulla Khel (lying between Khyber Pakhtunkhwa and Punjab Provinces), Pakistan. American Journal of Plant Sciences. 4: 98.

Shah GM, Khan MA. 2006. Common medicinal folk recipes of siran valley, Mansehra, Pakistan. Ethnobotanical Leaflet. 10:49-62.

Sheela CG, Augusti KT. 1992. Antidiabetic effects of $\mathrm{S}$-allyl cysteine sulphoxide isolated from garlic Allium sativum Linn. Indian Journal of Experimental Biology. 30:523-526.

Sher H, Hussain F. 2009. Ethnobotanical evaluation of some plant resources in Northern part of Pakistan. African Journal of Biotechnology. 8(17):4066-4076.

Shera AS, Basit A, Fawwad A, Hakeem R, Ahmedani MY, Hydrie MZI, Khwaja IA. 2010. Pakistan National Diabetes Survey: prevalence of glucose intolerance and associated factors in the Punjab Province of Pakistan. Primary care diabetes. 4(2), pp.79-83.

Shinde VS, Borkar AS, Badwaik RT. 2014. Evaluation and comparative study of hypoglycemic activity of aloe Barbadensis Miller with oral hypoglycemic drugs (glibenclamide and metformin) in rats. International Journal of Medical Research and Pharmaceutical Sciences. 4:31-36.

Shinkafi TS, Bello L, Hassan SW, Ali S. 2015. An ethnobotanical survey of antidiabetic plants used by
Hausa-Fulani tribes in Sokoto, Northwest Nigeria. Journal of Ethnopharmacology. 172:91-99.

Siddique A, Akhtar N, Khan MS, Anwar M, Samin J, Khan WM. 2016. Diversity, distribution and indigenous uses of the medicinal plants of district Karak, Khyber Pakhtunkhwa, Pakistan. Pakistan Journal of Weed Science Research. 22(2): 317-327. Tag H, Kalita P, Dwivedi P, Das AK, Namsa ND. 2012. Herbal medicines used in the treatment of diabetes mellitus in Arunachal Himalaya, northeast, India. Journal of Ethnopharmacology. 141:786-795.

Tahraoui A, El-Hilaly J, Israili ZH, Lyoussi B. 2007. Ethnopharmacological survey of plants used in the traditional treatment of hypertension and diabetes in south-eastern Morocco (Errachidia province). Journal of Ethnopharmacology. 110:105-117.

Tareen RB, Bibi T, Khan MA, Ahmad M, Zafar M, Hina S. 2010. Indigenous knowledge of folk medicine by the women of Kalat and Khuzdar regions of Balochistan, Pakistan. Pak J Bot. 42:1465-1485.

Tiwari JK, Dangwal LR, Rana CS, Tiwari P, Ballabha R. 2010. Indigenous uses of plant species in Nanda Devi Biosphere Reserve, Uttarakhand, India. Report and Opinion. 2:58-61.

Torres MP, Rachagani S, Purohit V, Pandey P, Joshi $S$, Moore ED, Johansson SL, Singh PK, Ganti AK, Batra SK. 2012. Graviola: a novel promising naturalderived drug that inhibits tumorigenicity and metastasis of pancreatic cancer cells in vitro and in vivo through altering cell metabolism. Cancer Letters. 323:29-40.

Trojan-Rodrigues M, Alves TLS, Soares GLG, Ritter MR. 2012. Plants used as antidiabetics in popular medicine in Rio Grande do Sul, southern Brazil. Journal of Ethnopharmacology. 139:155-163.

Ullah M, Khan MU, Mahmood A, Malik RN, Hussain M, Wazir SM, Daud M, Shinwari ZK. 2013. An ethnobotanical survey of indigenous medicinal plants in Wana district south Waziristan agency, Pakistan. Journal of Ethnopharmacology. 150(3):918-24.

Ullah R, Iqbal ZHZ, Hussain J, Khan FU, Khan N, Muhammad Z, Ayaz S, Ahmad S, Rehman NU, Hussain I. 2010. Traditional uses of medicinal plants in Darra Adam Khel NWFP Pakistan. Journal Medicinal Plants Research. 4:1815-1821.

Vinaykumar T, Eswarkumar K, Roy H. Evaluation of Antihyperglycemic Activity of Citrullus Colocynthis Fruit Pulp in Streptozotocin Induced Diabetic Rats. International Journal of Pharma Research and Health Sciences 4 (2) 1136-1142.

Yemele MD, Telefo PB, Lienou LL, Tagne SR, Fodouop CSP, Goka CS, Lemfack MC, Moundipa FP. 2015. Ethnobotanical survey of medicinal plants used for pregnant women' $s$ health conditions in Menoua division-West Cameroon. Journal of Ethnopharmacology. 160:14-31. 


\section{Annex}

\section{Questionnaire for collecting antidiabetic plant data}

\begin{tabular}{|l|l|}
\hline A. Informant details \\
\hline 1. Name: \\
\hline 2. Gender: \\
\hline 3 Ages: \\
\hline 4 Occupations: \\
\hline 5. Location/Residence: \\
\hline B. Informant knowledge about antidiabetic & \\
plants \\
\hline $\begin{array}{l}\text { 1. Name the plants which are used for treatment } \\
\text { of diabetes in the area? }\end{array}$ \\
\hline 2. Which part of the plants is used in \\
preparation of remedies? \\
\hline 3. How the plant is formulated? \\
\hline 4. How the plant is used? \\
\hline 5. How you collect the plants and store their \\
recipes?
\end{tabular}

